\title{
Co-precipitación de ettringita de rápida y lenta formación. Consecuencia: Efecto Sinérgico Expansivo. Su demostración mediante morteros y hormigones
}

\section{Co-precipitation of ettringite of rapid and slow formation. Consequence: Expansive Synergic Effect. Its demonstration by mortars and concretes}

\author{
R. Talero ${ }^{(*)}$
}

Recepción/Received: 12-III-09

Aceptación/Accepted: 25-X-10

RESUMEN

En investigaciones anteriores se ha demostrado que bastantes puzolanas pueden originar ettringita de rápida formación (de su $\mathrm{Al}_{2} \mathrm{O}_{3}{ }^{\mathrm{r}-}$ ) cuya velocidad es mayor que la de la ettringita de lenta formación, o de origen CPO (de su $\mathrm{C}_{3} \mathrm{~A}$ ). En este contexto: ¿qué tipo de efecto será el que produzcan finalmente ambas ettringitas?, ¿adición?, ¿sinergismo?, ¿antagonismo? o ¿inversión de la acción expansiva?

Para responder a estas cuestiones, 4 CPO y 12 cementos con $20 \%$, 30\% y $40 \%$ de metakaolín fueron ensayados mediante los métodos ASTM C 452-68, EN 196-1 y $\mathrm{RT}-86: \Delta \mathrm{L}$, y también mediante hormigones.

Los resultados obtenidos han demostrado que la precipitación conjunta en un medio selenitoso común, de ettringita de origen puzolana y de origen CPO, es siempre cuantitativamente hablando, más que aditiva, sinérgica, pudiendo ser por ello las consecuencias técnicas del Efecto Sinérgico Expansivo, beneficiosas, adversas o indiferentes, según que el exceso de sulfatos presentes sea más o menos adecuado.

Palabras clave: Ataque del yeso, Metakaolin, cementos Pórtland, ettringitas de "rápida" y "lenta" formación, sinergias.

\section{SUMMARY}

Several prior papers have shown that enough pozzolans can bring about rapid formation ettringite (from its $\mathrm{Al}_{2} \mathrm{O}_{3}{ }^{r^{-}}$). It has likewise been found that the formation rate of this ettringite is higher than the of slower forming ettringite originating from $O P C$ (from its $C_{3} A$ ). In this context: What type of effect will they ultimately produce? Addition? Synergism? Antagonism? or perhaps Inversion of final expansive action?

To reply to these questions, 4 PC and 12 blended cements containing $20 \%, 30 \%$ or $40 \%$ metakaolin, were tested using the ASTM C 452-68, EN 196-1 and RT-86: $\Delta L$ tests and also concrete specimens.

The experimental results have shows that the joint precipitation in a common sulfate medium, of ettringite from pozzolan and from OPC, was always more synergic than additive, and the technical consequences of the Expansive Synergic Effect may be classified as beneficial, adverse or indifferent according to its sulfates content in excess is more or less adequate.

Keywords: Gypsum attack, Metakaolin, Portland cements, "Rapid" and "slow" forming ettringites, synergies.

\footnotetext{
(*) Instituto de Ciencias de la Construcción Eduardo Torroja (IET-CSIC) (Madrid, España).
} 


\section{INTRODUCCIÓN}

En varias publicaciones anteriores sobre este tema de las ettringitas, se ha logrado demostrar mediante DRX (1-9) y SEM (2)(4-8)(10,11) y los métodos de ensayo Le Chatelier-Ansttet $(1,4,5,6,8,12,13)$, ASTM C 452-68 $(1,7,9,12,14)$ y $R T-86: \Delta L(1,9,12)$ (el mismo que el ensayo ASTM C 452-68 (15), sólo que con 21,0\% SO en su pasta cementante en lugar de $7,0 \%$ ), que casi todas las adiciones puzolánicas pueden formar ettringita de rápida formación, ett-rf (1-11), en función de su contenido de alúmina reactiva, $\mathrm{Al}_{2} \mathrm{O}_{3}{ }^{\mathrm{r}}$, vítrea y/o amorfa o alúmina tetra- o penta-coordinada (16), cuya $\mathbf{V}_{\mathbf{f}}$ es mayor que la de la ettringita de lenta formación, ett-If * (1-11), o de origen $\mathrm{C}_{3} \mathrm{~A}$ de los cementos Pórtland ordinarios, CPO, y como consecuencia lógica, su tamaño es $\approx 10$ veces menor $(4-8,10)$. Lo que en absoluto menoscaba su característica expansividad al formarse sino más bien todo lo contrario, es decir, que la aumenta, y aún menos que lo que debería porque su volumen molar se ha considerado equivalente al de la $\gamma-\mathrm{Al}_{2} \mathrm{O}_{3}$ lo que no es correcto. De aquí que todos los métodos de ensayo referidos hayan puesto de manifiesto además, que ninguno de los cementos de mezcla, PUZC, que se prepararon con metakaolin (MK), pudo ser calificado de elevada ni modera resistencia sulfática, RS $(1,4-11,14)$. Dado que dicha puzolana debe poseer casi toda su alúmina en forma reactiva, $\mathrm{Al}_{2} \mathrm{O}_{3}{ }^{\mathrm{r}-}$, o al menos, la mayor parte $(4,9)$, motivo por el cual, todas sus probetas de pasta, mortero y hormigón son degradadas por ataque del yeso mucho más rápidamente que las de su correspondiente CPO puro $(2,4,8,10)$. Lo que justificó haberle podido adscribir el calificativo de "ataque rápido del yeso" $(1,4,5,10,12)$.

Por consiguiente y de acuerdo con todo lo anterior, al poder ser ambos tipos de ettringitas

- de orígenes diferentes: la ett-rf o de origen $\mathrm{Al}_{2} \mathrm{O}_{3}{ }^{\mathrm{r}-}$ de MK en esta caso, y la ett-If o de origen $\mathrm{C}_{3} \mathrm{~A}$ de los $\mathrm{CPO}, \mathrm{y}$

- de etiologías también diferentes: velocidades de formación y cantidades formadas, diferentes, y

al llevar implícita su génesis un proceso

- competitivo diverso, por reaccionar con el yeso, y

- expansivo y disruptivo también diversos,

* Ettringita que se forma más lentamente del $C_{3} A$ presente en los CPO después de su hidratación inicial. No se intenta que el término ett-If $(1,4-11,13)$ signifique que este tipo de ettringita sea siempre el producto de reacción de lenta formación, cuando co-precipita con ett-rf $(1,4-11)$, sino únicamente que en esta última circunstancia, se forma del $\mathrm{C}_{3} \mathrm{~A}(\%)$ de los $\mathrm{CPO}$.

\section{INTRODUCTION}

In several previous publications on this matter of ettringites, it has been shown by XRD (1-9) and SEM (2)(4-8)(10,11) and by Le Chatelier-Ansttet, (L-A) (1, 4, $5,6-8,12,13), A S T M C 452-68(1,7,9,12,14)$ and $R T-86: \Delta L(1,9,12)$ (similar to ASTM C 452-68 (15) but the cement with $21.0 \% \mathrm{SO}_{3}$ instead of $7.0 \% \mathrm{SO}_{3}$ ) tests, that almost all pozzolanic additions can form ettringite of rapid formation, ett-rf (1-11), in relation to their content of reactive alumina, $\mathrm{Al}_{2} \mathrm{O}_{3}{ }^{r-}$ (vitreous or amorphous or tetra- or penta-coordinated alumina (16)), and that the $V_{\boldsymbol{f}}$ of this is greater than that of the slow formation ettringite, ett-If ${ }^{*}(1-11)$, or from $C_{3} A$ origin of the CPO, and as a logical consequence, ett-rf is $\approx 10$ times smaller (4-8, 10). This does not at all diminish its expansive characteristic on formation but rather has the opposite effect, it increases it, and even less than it should have done, because the molecular volume of this $\mathrm{Al}_{2} \mathrm{O}_{3}{ }^{r-}$ is only considered to be equivalent to that of the $\gamma$-alumina, which is not the correct. All the test methods and techniques referred to here have demonstrated that none of the blended cements (POZC) prepared with metakaolin (MK) could be qualified as having high or even moderate sulfate resistance $(1,4-11,14)$. Since almost all the alumina present in this $M K$, or at least the greater part, must be regarded as being "reactive", $\mathrm{Al}_{2} \mathrm{O}_{3}{ }^{r-}(4,9)$, and for this reason, all its $\mathrm{POZC}$ pastes and mortars and concretes specimens would be damaged by gypsum attack much more quickly than the plain $\operatorname{OPC}(2,4,8,10)$. This detrimental effect derived from gypsum attack was to such an extent and rapid, that it could be described as "rapid gypsum attack" $(1,4,5,10,12)$.

Therefore, in accordance with the foregoing discussion, since the ettringites may be of different origins and etiologies (different formation rates, sizes and quantities) and since, their precipitation involves a process that is, diversely competitive, because of its reaction with gypsum, and also diversely expansive and disruptive, when the two are formed together in a sulfate-bearing medium such as concrete, mortar, or paste made with these POZCs attacked by plaster-bearing solutions, the key questions are: What will the outcome of the expansive effects be? What type of effect will they ultimately produce? addition? synergism? antagonism? or perhaps inversion of final expansive action?

\footnotetext{
* Ettringite that forms much more slowly from $C_{3} A$ present in OPC, after its initial hydration. Tthe term ett-If (1, 4-11, 13 ) is not intended to mean that this type of ettringite is always necessarily the product of slow formation when co-precipitating with ett-rf $(1,4-11)$, but merely that in the latter circumstance, it is formed from the $C_{3} A(\%)$ content present in OPC.
} 
al formarse ambas conjuntamente o al unísono, en un medio selenitoso común, como puede ser el de un hormigón, mortero o pasta de tales PUZC atacados por aguas selenitosas, cabe preguntarse con fundamento finalmente:

- ¿Cómo serán sus efectos expansivos resultantes...?

- ¿En qué se traducirán finalmente...?, ¿en una adición...?, ¿sinergismo...? ¿antagonismo...?, o quizás... ¿en una inversión de la acción expansiva resultante final...?

No importa que la respuesta haya sido dada ya, en el título de este artículo y en el segundo párrafo de este mismo apartado. Porque lo realmente importante es demostrarlo, pero sobre todo, justificarlo científica y técnicamente, lo que será objeto de otro artículo, es decir,

- ¿Cómo serán las implicaciones prácticas de la precipitación conjunta -co-precipitación- de los dos tipos de ettringita para la durabilidad del hormigón que las contiene, ya sea éste atacado o no por aguas selenitosas (sulfatos), o por aguas con sales de deshielo (cloruros),o por agua de mar (cloruros, sulfatos, magnesio, etc.), y/o por el $\mathrm{CO}_{2}$ del aire (carbonatación), o por áridos potencialmente reactivos frente a los álcalis del cemento (reactividad áridoálcali), o por retracción térmica (debido al desprendimiento excesivo de calor de hidratación liberado que provoca su precipitación conjunta -co-precipitación-?).

- ¿Serán todas esas consecuencias tecnológicas positivas?, o ¿serán todas ellas negativas?, o ¿serán positivas unas... y negativas otras....? y si este último es el caso... ¿cuáles serían positivas de todas ellas... y cuáles otras serían negativas...?

Naturalmente, las respuestas a todas esas preguntas rebasan los límites de este artículo. Por lo que se impuso la necesidad de tener que contestarlas en otras publicaciones o artículos que ya han sido publicados (1-11, $14,17-33)$, o se van a publicar próximamente (11), habiendo sido además todos ellos parte integrante de tesis doctorales $(1,18,22)$.

No obstante, a algunas otras de esas preguntas se les ha logrado dar respuesta en este artículo, y más concretamente, a todas aquéllas que están relacionadas con su demostración, pudiéndose ver al final del mismo en el apartado 5. CONCLUSIONES. Al resto, relacionadas más bien con su explicación y justificación y consecuencias técnicas, que son más importantes e interesantes todavía, se les dará también respuesta en próximos artículos.
The fact that the answer to these questions is once again implicit in the title of this paper and in the second paragraph of this same section is irrelevant.

Henceforth, the primary concern should be to actually prove those assertions and their practical implications -which are the main objective of this study-, and above all, to explain and justify them, which will be aim for a future paper. Nevertheless, the questions relating to the practical implications can already be formulated here, namely:

- What would the practical implications of the joint precipitation (co-precipitation) of the two types of ettringite be for the durability of the cement concrete in which their respective $\mathrm{Al}_{2} \mathrm{O}_{3}{ }^{r-}$ and $\mathrm{C}_{3} \mathrm{~A}$ origins may be potentially contained, whether or not it is attacked by a plaster-bearing solution, water from thawed ice (chlorides), seawater (chlorides, sulfates, magnesium, etc.) and/or atmospheric $\mathrm{CO}_{2}$ (carbonation), aggregates potentially reactive with cement alkalis (alkali-silica reaction), thermal shrinkage (owing to excess release, or otherwise, of hydration heat due to its joint precipitation (co-precipitation))?

- Would all these practical implications be beneficial? adverse? some beneficial and others adverse? In the latter case, which would be beneficial and which adverse?

Naturally, the answers to all these questions fall outside the limits of this paper and must necessarily be tackled in other subsequent studies. Some have been already been published $(1-11,14,17-33)$ and the rest of them are expected to be published in a near future (11), forming part of $P h D$ dissertations that are closed to their realization $(1,18,22)$.

Nevertheless, some of these issues and questions have been addressed in this study as it can be observed from the Section 5. CONCLUSIONS, but the rest of them, more related with their explanation and justification (which are much more important yet) and technical consequences as well, will be all addressed in future papers. 


\section{OBJETIVOS}

Los objetivos de este estudio han sido por tanto los siguientes:

1. Cuantificar mediante diferentes parámetros químicos, físicos y mecánico-resistentes, el efecto expansivo resultante de la formación conjunta (co-precipitación) de la ett-rf y la ett-If en un medio selenitoso común: probetas de mortero y hormigón de cemento con exceso de yeso (15,05\%).

2. En función de la cuantificación anterior, determinar si dicho efecto expansivo o no resultante, según el parámetro físico o mecánico que se considere, desemboca o se traduce para bien, para mal, o para peor, en una adición, sinergismo, antagonismo o inversión de la acción expansiva. Así y mediante este segundo objetivo, el efecto final resultante de la formación conjunta (co-precipitación) en un medio selenitoso común, de la ett-rf y la ett-If, podrá ser denominado en lo sucesivo, con la propiedad debida.

3. Determinar si las consecuencias finales del objetivo 2 , resultante entre los dos tipos de ettringita, se puede estimar que no sea adversa $(1,4,5,10,14)$, sino beneficiosa y cómo.

\section{PARTE EXPERIMENTAL}

\subsection{Materiales de partida}

Los materiales utilizados en esta investigación se encuentran en la Tabla 1. De acuerdo con el diagrama ternario de Eitel (34), se tuvieron que elegir los siguientes materiales:

1. Dos CPO -P-1 y P-2 (P-n $\left.n^{0}\right)$ - y dos CPRS -PY-4 y PY-6 (PY-no)-.

2. Metakaolín (MK) que se preparó calcinando caolín (con un contenido de $\approx 50 \%$ de cuarzo) a $750{ }^{\circ} \mathrm{C}$, durante el tiempo necesario, siendo además su composición granulométrica acorde con la norma ASTM C 595-95 (35) [cantidad máxima retenida por tamizado en húmedo, en el tamiz no $325(45 \mu \mathrm{m})=$ 20\%].

3. Yeso: se utilizó como agresivo piedra de yeso natural molida, con un elevado contenido de $\mathrm{CaSO}_{4} \cdot 2 \mathrm{H}_{2} \mathrm{O}$ (Tabla 1).

El metakaolín (MK) es una puzolana artificial que está siendo muy estudiada últimamente, dado su interés como material de construcción, mereciendo especial atención los artículos (36-44) por estar relacionados de una manera u otra con este estudio.

\section{OBJECTIVES}

The objectives of this study reported here were as follows:

1. To quantify the performance of the joint precipitation (co-precipitation) of ett-rf and ett-If in a common plaster-bearing solution, using different chemical, physical and mechanical strength parameters determined to cement concretes and new mortars, with excess of gypsum (15.05\%).

2. To determine, on the grounds of quantitative findings, whether the resulting performance can be considered to constitute Addition, Synergism, Antagonism or Inversion of final expansive action. Thus from now on, the latter of these two objectives will make it possible to appropriately denominate the result of such joint formation (co-precipitation) of ettrf and ett-lf.

3. To determine if the final consequences of the objective 2, resulting between the two types of ettringites, can once again be esteemed not to be adverse $(1,4,5,10,14)$ but beneficial, and how.

\section{EXPERIMENTAL}

\subsection{Starting materials}

The materials used in this research are shown in Table 1. Pursuant to Eitel's ternary diagram (34), the following materials were chosen:

1. Two OPC $-P-1$ and $P-2\left(P-n^{\circ}.\right)-$ and two SRPC $-P Y-4$ and $P Y-6\left(P Y-n^{0}.\right)$ -

2. Metakaolin (MK) was prepared by calcinating kaolin (with $\approx 50 \%$ quartz content) at $750{ }^{\circ} \mathrm{C}$, graded to standard ASTM C 595-95 (35) (maximum 20\% retained when wet-sieved through sieve No. 325 $(45 \mu m))$.

3. Natural stone gypsum (with a high $\mathrm{CaSO}_{4} \cdot 2 \mathrm{H}_{2} \mathrm{O}$ content) was used as the aggressive medium (Table 1).

Although metakaolin has been extensively studied in the literature in light of the value of this artificial pozzolan as a building material, particular attention is drawn here to the papers (36-44) that are related in one way or another to this study. 
Tabla 1 / Table 1

Determinaciones químico-físicas de los materiales cementíceos. Chemical-Physic determinations of cementing materials.

\begin{tabular}{|c|c|c|c|c|c|c|c|c|c|}
\hline \multirow{3}{*}{$\begin{array}{l}\text { Parámetros químicos I } \\
\text { Chemical Parameters (\%) }\end{array}$} & \multicolumn{4}{|c|}{$\begin{array}{l}\text { Cementos Portland I } \\
\text { Portland Cements }\end{array}$} & \multirow{3}{*}{$\begin{array}{c}\begin{array}{c}\text { Puzolana / } \\
\text { Pozzolan }\end{array} \\
\text { MK } \\
{[2,55]}\end{array}$} & \multicolumn{4}{|c|}{ Yeso / Gypsum } \\
\hline & \multicolumn{2}{|c|}{ CPO / OPC } & \multicolumn{2}{|c|}{ CPRS / SRPC } & & \multirow{2}{*}{\multicolumn{2}{|c|}{$\begin{array}{l}\text { Parámetros químicos I } \\
\text { Chemical Parameters (\%) }\end{array}$}} & \multirow{2}{*}{\multicolumn{2}{|c|}{$\begin{array}{l}\text { Composición mineralógica I } \\
\text { Mineralogical Composition } \\
\text { (\%) }\end{array}$}} \\
\hline & $\begin{array}{c}\mathrm{P}-1 \\
{[3.08]^{* 2}}\end{array}$ & $\begin{array}{c}\text { P-2 } \\
{[3.06]}\end{array}$ & $\begin{array}{l}\text { PY-4 } \\
{[3.16]}\end{array}$ & $\begin{array}{l}\text { PY-6 } \\
{[3.21]}\end{array}$ & & & & & \\
\hline P.F. / L.O.I. & 1.60 & 2.91 & 1.64 & 1.11 & 0.60 & \multirow{2}{*}{$\begin{array}{l}\mathrm{H}_{2} \mathrm{O}\left(40^{\circ} \mathrm{C} \text { to }\right. \\
\left.217^{\circ} \mathrm{C}\right)\end{array}$} & \multirow{2}{*}{20.13} & \multirow{2}{*}{$\mathrm{CaSO}_{4} 2 \mathrm{H}_{2} \mathrm{O}$} & \multirow{2}{*}{95,58} \\
\hline R.I., / I.R. ${ }^{*}$ & 0.70 & 1.21 & 0.43 & 0.15 & 0.22 & & & & \\
\hline $\mathrm{SiO}_{2}$ & 19.18 & 19.36 & 22.10 & 21.70 & 73.53 & \multirow{2}{*}{$\begin{array}{c}\mathrm{CO}_{2}\left(217^{\circ} \mathrm{C} \text { to }\right. \\
\left.1000{ }^{\circ} \mathrm{C}\right)\end{array}$} & \multirow{2}{*}{0.75} & \multirow{3}{*}{$\begin{array}{l}\mathrm{CaSO}_{4}{ }_{1}^{1} / 2 \mathrm{H}_{2} \mathrm{O} \text { y/o } \\
\text { and/or } \mathrm{CaSO}_{4}\end{array}$} & \multirow{3}{*}{2,47} \\
\hline $\mathrm{Al}_{2} \mathrm{O}_{3}$ & 6.44 & 6.03 & 1.98 & 1.52 & 23.11 & & & & \\
\hline $\mathrm{Fe}_{2} \mathrm{O}_{3}$ & 1.75 & 2.89 & 4.46 & 4.11 & 1.19 & R.I. / I.R. ${ }^{*}$ & 0.26 & & \\
\hline $\mathrm{CaO}$ & 63.94 & 59.49 & 65.59 & 67.97 & 0.63 & $\mathrm{SiO}_{2}$ & 0.04 & & \\
\hline MgO & 1.48 & 1.21 & 0.83 & 0.42 & 0.03 & $\mathrm{SO}_{3}$ & 45.87 & $\mathrm{CaCO}_{3}$ & 0,75 \\
\hline $\mathrm{Na}_{2} \mathrm{O}$ & 0.90 & 1.23 & 0.15 & 0.43 & 0.07 & $\mathrm{CaO}$ & 32.54 & $\mathrm{MgCO}_{3}$ & 0,81 \\
\hline $\mathrm{K}_{2} \mathrm{O}$ & 0.52 & 0.69 & 0.05 & 0.20 & 0.70 & $\mathrm{MgO}$ & 0.36 & TOTAL & 99.61 \\
\hline $\mathrm{SO}_{3}$ & 3.50 & 4.94 & 2.78 & 2.34 & 0.00 & $\mathrm{Na}_{2} \mathrm{O}$ & 0.02 & \multirow{4}{*}{$\mathrm{H}_{2} \mathrm{O}$ at $40{ }^{\circ} \mathrm{C}$} & \multirow{4}{*}{0,41} \\
\hline TOTAL & 100.01 & 99.96 & 100.01 & 99.50 & 100.05 & $\mathrm{~K}_{2} \mathrm{O}$ & 0.01 & & \\
\hline $\mathrm{H}_{2} \mathrm{O}\left(105^{\circ} \mathrm{C}\right)$ & 0.24 & 0.93 & 0.22 & 0.22 & 100.05 & TOTAL & 99.98 & & \\
\hline CaO libre / free & 1.9 & 0.7 & 1.2 & 1.75 & - & & & & \\
\hline $\begin{array}{l}\text { Composición mineralógica I } \\
\text { Mineralogical Composition } \\
\text { (\%) }\end{array}$ & \multicolumn{4}{|c|}{$\begin{array}{l}\text { Cálculo potencial de bogue I } \\
\text { Bogue Potential Calculus }\end{array}$} & & & & & \\
\hline $\mathrm{C}_{3} \mathrm{~S}$ & 51.05 & 33.47 & 58.19 & 79.43 & & & & & \\
\hline $\mathrm{C}_{2} \mathrm{~S}$ & 16.48 & 30.26 & 19.46 & 2.29 & & & & & \\
\hline $\mathrm{C}_{3} \mathrm{~A}$ & 14.11 & 11.09 & 0.00 & 0.00 & & & & & \\
\hline $\mathrm{C}_{4} \mathrm{AF}\left(+\mathrm{C}_{2} \mathrm{~F}\right) \mathrm{ss}$ & 5.33 & 8.79 & 11.75 & 10.19 & & & & & \\
\hline $\mathrm{C}_{4} \mathrm{AF}+2 \mathrm{C}_{3} \mathrm{~A}$ & 33.55 & 30.97 & 11.75 & 10.19 & & & & & \\
\hline $\mathrm{C}_{4} \mathrm{AF}+\mathrm{C}_{3} \mathrm{~A}$ & 19.44 & 19.88 & 11,75 & 10,19 & MK & \multirow{2}{*}{\multicolumn{4}{|c|}{$\begin{array}{l}{ }^{*}=\text { Residuo insoluble } / \text { Insoluble Residue } \\
{ }^{*} \mathbf{2}=\text { Pesos específico } / \text { Specific gravity }\left(\mathrm{g} / \mathrm{cm}^{3}\right) \\
\text { MK }=\text { Metakaolín (con / with } \approx 50,0 \% \text { de cuarzo / quartz content) }\end{array}$}} \\
\hline SEB / BSS $\left(m^{2} / \mathbf{k g}\right)$ & 319 & 302 & 323 & 329 & 398 & & & & \\
\hline
\end{tabular}

\subsection{Procedimiento experimental}

En primer lugar, se prepararon 12 cementos de mezcla (PUZC) con los cuatro cementos Pórtland (CP) anteriores y el $\mathrm{MK}$, en las proporciones de mezcla $80 / 20 \%, 70 / 30 \%$ y $60 / 40 \%$. La proporción $100 / 00 \%(=100 / 00)$ corresponde al CPO $\left(=\mathrm{P}-n^{\circ}\right)$ ó CPRS $\left(=\mathrm{PY}-\mathrm{n}^{\circ}\right)$ puro.

En segundo lugar, todos esos PUZC se analizaron mediante el ensayo de Frattini (45) al objeto de confirmar sus características puzolánicas a 2, 7 y 28 días, más tarde relacionadas con el ataque de los sulfatos (Figura 1), En paralelo, a algunos de esos CP y PUZC se les determinó también su cantidad de agua de consistencia normal, estabilidad de volumen (46), tiempos de fraguado (Tabla 2) (46) y resistencias mecánicas (Tabla 3) (47) de sus morteros tipo ASTM C 452-68 (15) y EN 196-1 (47).

En tercer lugar, cada CP puro y sus PUZC fueron ensayados según el método ASTM C 452-68 (15), habiéndoseles medido los siguientes parámetros a sus correspondientes probetas de 1 "x1"x111/4": Incremento de longitud, $\Delta \mathrm{L}(\%)$

\subsection{Operating procedure}

First of all, 12 blended cements or POZC, with ratios of $80 / 20 \%, 70 / 30 \%$ and $60 / 40 \%$ (\%P-n\% $\% M K$ or $\% P Y$ n०\%MK) were prepared with the 4 previous $P C$ and the MK. A ratio of $100 / 00 \%$ or $100 / 00$ is indicative of plain $O P C\left(=P-n^{\circ}\right)$ or plain SRPC $\left(=P Y-n^{\circ}\right)$.

Secondly, all these POZC were then analyzed by the Frattini test (45) to determine their pozzolanic properties at 7 and 28 days (later related to sulfate attack) (Figure 1). In parallel, normal consistencies, volume stability (Le Chatelier's needles) (46), setting times (Table 2) (46) and mechanical strengths (MS) (Table 3) (47) of their mortars ASTM C 452-68 (15) and EN 196-1 (47) types from some $P C$ and their POZC were determined as well.

Thirdly, PC and POZC were tested using the ASTM C 45268 (15) procedure. Only four specimens (1"x1"x111/4") were made from each $P C$ and $P O Z C$ to determine length increases, $\boldsymbol{\Delta L}(\%)$ (Figures $2(a, b))$, although three 


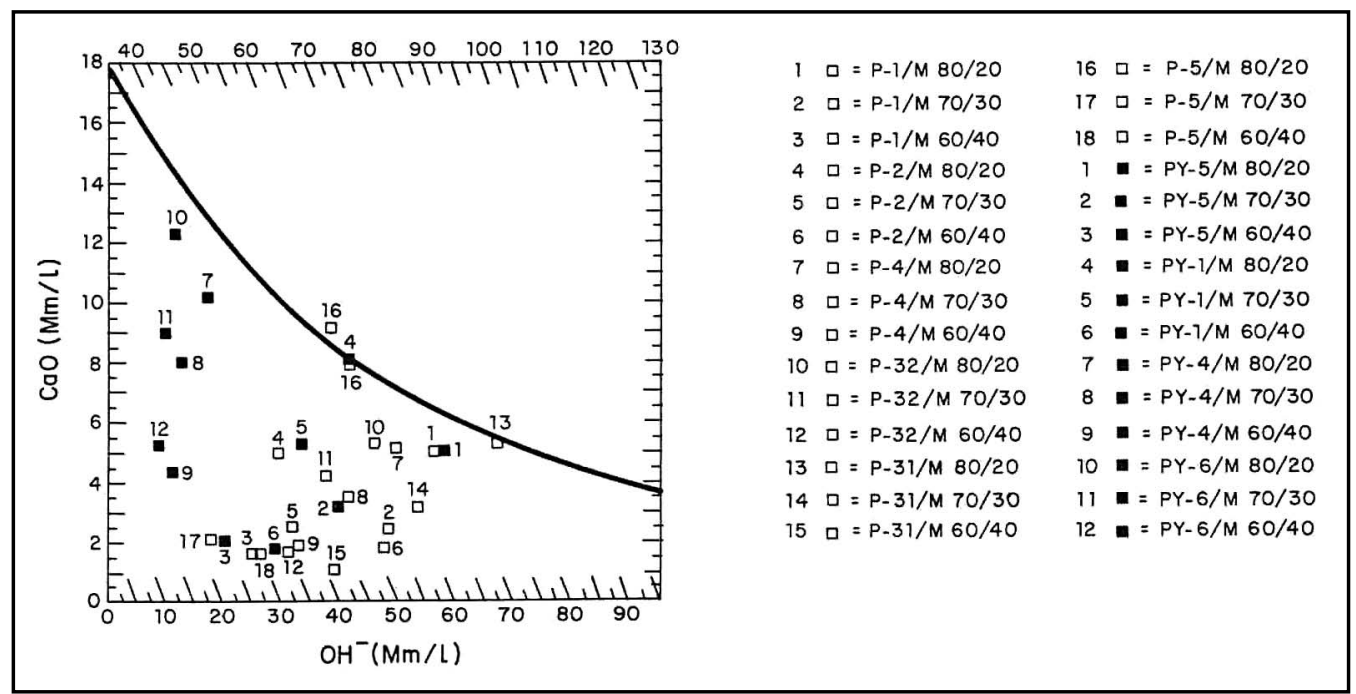

Figura 1. Ensayo de Frattini de los 30 PUZC. Resultados a 28 y/o 7 días. $M=M K$.

Figure 1. Frattini test of the 30 POZC. Results at 28 and/or 7 days. $M=M K$.

(Figuras $2(\mathrm{a}, \mathrm{b})$ ) y velocidad de crecimiento longitudinal, $\mathrm{Vcl}(=\Delta \mathrm{L}(\%) /$ día) (Figuras $3(\mathrm{a}, \mathrm{b}, \mathrm{c}, \mathrm{d}))$. El contenido de sulfatos del agua de conservación, $\left\{\mathrm{SO}_{4}{ }^{2-}\right\}_{\text {lcp }}$ de estas probetas también fue determinado, precipitándolos como $\mathrm{BaSO}_{4}$ en medio $\mathrm{ClH}$ (Figuras 2 (c, d, e) y Tabla 4). Los further specimens were prepared as necessary to ratify any questionable previous $\boldsymbol{\Delta L}(\%)$ values (the leftover mortar amount was practically always the same). Length, $\boldsymbol{\Delta} \mathbf{L}_{\mathbf{x d}}(\%)$, was measured at $1,7,14,21,28,60$, 90 days, or even later, depending on the aim pursued.

Tabla 2 / Table 2

Consistencia normal, tiempos de fraguado y estabilidades de volumen de varios CP y sus PUZC con MK. Normal consistency, times of setting and volume stability of several PC and their POZC with MK.

\begin{tabular}{|c|c|c|c|c|c|c|c|c|c|c|c|c|c|c|}
\hline \multirow{4}{*}{\multicolumn{3}{|c|}{ Cementos / Cements }} & \multirow{5}{*}{ 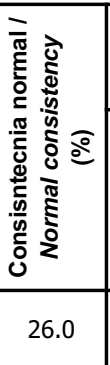 } & \multirow{2}{*}{\multicolumn{3}{|c|}{$\begin{array}{l}\text { Tiempos de fraguado I } \\
\text { Times of Setting } \\
\text { (h:min) }\end{array}$}} & \multicolumn{8}{|c|}{ Agujas Le Chatelier / Le Chatelier Needles (mm) } \\
\hline & & & & & & & \multicolumn{8}{|c|}{ Agua / Water (días / days) } \\
\hline & & & & \multirow{2}{*}{$\begin{array}{c}\text { Inicial / } \\
\text { Initial }\end{array}$} & \multirow{2}{*}{ Final } & \multirow{2}{*}{$\begin{array}{l}\text { Tiempo de } \\
\text { fraguado I } \\
\text { Setting } \\
\text { Time }\end{array}$} & $\begin{array}{c}\text { Cal. I } \\
\text { Hot }\end{array}$ & \multicolumn{7}{|c|}{ Fría / Cold $\left(21 \pm 2{ }^{\circ} \mathrm{C}\right)$} \\
\hline & & & & & & & 7 & 7 & 14 & 21 & 28 & 60 & 90 & 120 \\
\hline \multirow{5}{*}{ 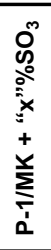 } & $\begin{array}{l}\text { Ch } \\
\operatorname{mi}^{\circ} \\
+\end{array}$ & $100 / 00$ & & 2:00 & $2: 39$ & $0: 39$ & 0.7 & 1.70 & 1.70 & 1.70 & 1.70 & 1.70 & 1.70 & 1.70 \\
\hline & \multirow{4}{*}{ 高 } & [100/00] & 32.0 & $3: 55$ & $6: 15$ & $2: 20$ & * & * & * & * & * & * & $*$ & * \\
\hline & & {$[80 / 20]$} & 29.6 & $3: 15$ & $4: 10$ & $0: 55$ & 0.5 & 3.33 & 5.65 & 7.20 & 9.35 & 12.00 & 12.15 & 12.15 \\
\hline & & [70/30] & 30.8 & 3:05 & $4: 10$ & 1:05 & 0.5 & 5.15 & 6.00 & 6.33 & 6.65 & 6.71 & 6.75 & 7.75 \\
\hline & & [60/40] & 32.8 & $3: 15$ & $4: 10$ & $0: 55$ & 0.5 & 5.75 & 5.75 & 5.75 & 5.75 & 5.75 & 5.75 & 5.75 \\
\hline \multirow{5}{*}{ 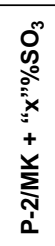 } & $\begin{array}{l}\stackrel{+}{+} \\
+ \\
+ \\
+\end{array}$ & $100 / 00$ & 24.0 & $2: 10$ & 3:09 & $0: 59$ & 0.8 & 2.60 & 2.60 & 2.60 & 2.60 & 2.60 & 2.60 & 2.60 \\
\hline & \multirow{4}{*}{$\begin{array}{l}\stackrel{\circ}{\circ} \\
+ \\
+\end{array}$} & {$[100 / 00]$} & 29.6 & $4: 25$ & $7: 35$ & $3: 10$ & $*$ & * & $*$ & $*$ & * & $*$ & * & * \\
\hline & & {$[80 / 20]$} & 28.7 & $4: 40$ & $7: 30$ & $2: 50$ & 0.5 & 5.83 & 8.16 & 10.00 & 11.50 & 15.33 & 16.33 & 16.33 \\
\hline & & {$[70 / 30]$} & 29.9 & $5: 35$ & $7: 55$ & $2: 20$ & 0.5 & 6.00 & 7.50 & 8.00 & 8.16 & 8.17 & 8.00 & 8.00 \\
\hline & & {$[60 / 40]$} & 32.2 & $4: 35$ & $7: 15$ & $2: 40$ & 0.5 & 7.33 & 7.33 & 7.33 & 7.33 & 7.33 & 7.33 & 7.33 \\
\hline \multirow{5}{*}{ 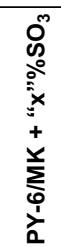 } & $\stackrel{\substack{\tilde{N} \\
+}}{\circ}$ & $100 / 00$ & 21.2 & 0:05 & $0: 25$ & $0: 20$ & 1.0 & 0.30 & 0.30 & 0.30 & 0.30 & 0.30 & 0.30 & 0.30 \\
\hline & \multirow{4}{*}{$\begin{array}{l}\text { ठें } \\
\text { ஸे } \\
+\end{array}$} & {$[100 / 00]$} & 28.0 & $4: 15$ & $6: 10$ & $1: 55$ & $*$ & $*$ & $*$ & $*$ & $*$ & $*$ & $*$ & $*$ \\
\hline & & {$[80 / 20]$} & 27.2 & $6: 15$ & $8: 10$ & $1: 55$ & 0.5 & 2.00 & 3.50 & 3.55 & 3.60 & 3.90 & 4.00 & 4.50 \\
\hline & & \begin{tabular}{|l}
{$[70 / 30]$} \\
\end{tabular} & 28.8 & $5: 45$ & $6: 55$ & $1: 10$ & 0.0 & 3.20 & 3.70 & 3.80 & 4.00 & 4.60 & 4.60 & 4.70 \\
\hline & & {$[60 / 40]$} & 31.6 & $5: 50$ & $7: 35$ & $1: 45$ & 0.5 & 5.00 & 5.00 & 5.00 & 5.00 & 5.00 & 5.00 & 5.00 \\
\hline
\end{tabular}

NOTA: El resto de cementos (CPO, CPRS y sus PUZC con MK) proporcionaron valores de similar orden de magnitud para cada parámetro. / NOTE: The rest of the cements (OPC, SRPC and their POZC with MK) reached similar order values for each parameter.

* Estas determinaciones no fueron necesarias para este estudio. / * These determinations were not necessary for this study. 
Tabla 3 / Table 3

Resistencias mecánicas de los CPO, P-1 y P-2, y del CPRS PY-6, y sus PUZC con MK. Mechanical strengths from OPC, $P-1$ and $P-2$, SRPC PY-6 and their POZC with MK.

\begin{tabular}{|c|c|c|c|c|c|c|c|c|c|}
\hline \multirow{4}{*}{\multicolumn{2}{|c|}{ Cementos / Cements }} & \multicolumn{8}{|c|}{$\begin{array}{l}\text { Resistencias mecánicas / Mechanical Strengths } \\
\text { Tipos de morteros de cemento / Cement mortar types: } \\
\text { EN 196-1 }\left(=-7.0 \% \mathrm{SO}_{3}\right) \text {; ASTM C452-68 }\left(=+7.0 \% \mathrm{SO}_{3}\right)\end{array}$} \\
\hline & & \multicolumn{4}{|c|}{ Flexotracción / Flexural strength, FS (MPa) } & \multicolumn{4}{|c|}{ Compresión / Compressive strength, CS (MPa) } \\
\hline & & \multicolumn{2}{|c|}{28 días / days } & \multicolumn{2}{|c|}{90 días / days } & \multicolumn{2}{|c|}{28 días / days } & \multicolumn{2}{|c|}{90 días / days } \\
\hline & & $-7.0 \% \mathrm{SO}_{3}$ & $+7.0 \% \mathrm{SO}_{3}$ & $-7.0 \% \mathrm{SO}_{3}$ & $+7.0 \% \mathrm{SO}_{3}$ & $-7.0 \% \mathrm{SO}_{3}$ & $+7.0 \% \mathrm{SO}_{3}$ & $-7.0 \% \mathrm{SO}_{3}$ & $+7.0 \% \mathrm{SO}_{3}$ \\
\hline P-1 & $100 / 00$ & 7.0 & 4.4 & 7.3 & 8.9 & 47.7 & 27.7 & 50.3 & 53.0 \\
\hline P-1/MK & $80 / 20$ & 8.2 & 9.7 & 8.4 & 10.3 & 54.9 & 40.1 & 55.9 & 39.3 \\
\hline $\mathrm{P}-1 / \mathrm{MK}$ & $70 / 30$ & 8.7 & 8.9 & 8.9 & 9.8 & 53.3 & 32.6 & 54.3 & 48.6 \\
\hline P-1/MK & $60 / 40$ & 8.6 & 8.5 & 8.8 & 8.9 & 48.3 & 43.6 & 49.2 & 45.7 \\
\hline P-2 & $100 / 00$ & 4.0 & 4.4 & 4.2 & 5.2 & 27.3 & 18.3 & 32.4 & 29.7 \\
\hline P-2/MK & $80 / 20$ & 8.2 & 9.8 & 8.4 & 10.5 & 60.8 & 38.0 & 61.9 & 43.6 \\
\hline P-2/MK & $70 / 30$ & 9.7 & 9.5 & 9.9 & 9.7 & 49.7 & 38.8 & 50.6 & 45.4 \\
\hline P-2/MK & $60 / 40$ & 7.7 & 8.6 & 7.9 & 8.9 & 45.7 & 41.1 & 46.5 & 43.3 \\
\hline PY-6 & $100 / 00$ & 7.8 & 6,1 & 7.3 & 7.4 & 54.3 & 30.9 & 63.0 & 32.7 \\
\hline PY-6/MK & $80 / 20$ & 8.8 & 6,5 & 9.8 & 9.3 & 59.8 & 47.3 & 71.6 & 54.5 \\
\hline PY-6/MK & $70 / 30$ & 9.5 & 9.2 & 10.4 & 11.0 & 57.2 & 32.1 & 68.4 & 47.8 \\
\hline PY-6/MK & $60 / 40$ & 7.3 & 10.1 & 8.1 & 10.7 & 54.3 & 39.3 & 54.6 & 46.4 \\
\hline
\end{tabular}

NOTA: El resto de cementos (CPRS PY-4 y sus POZC) proporcionaron valores de similar orden de magnitud para cada parámetro. NOTE: The rest of the cements (SRPC PY-4 and its POZC) reached similar order values for each parameter.

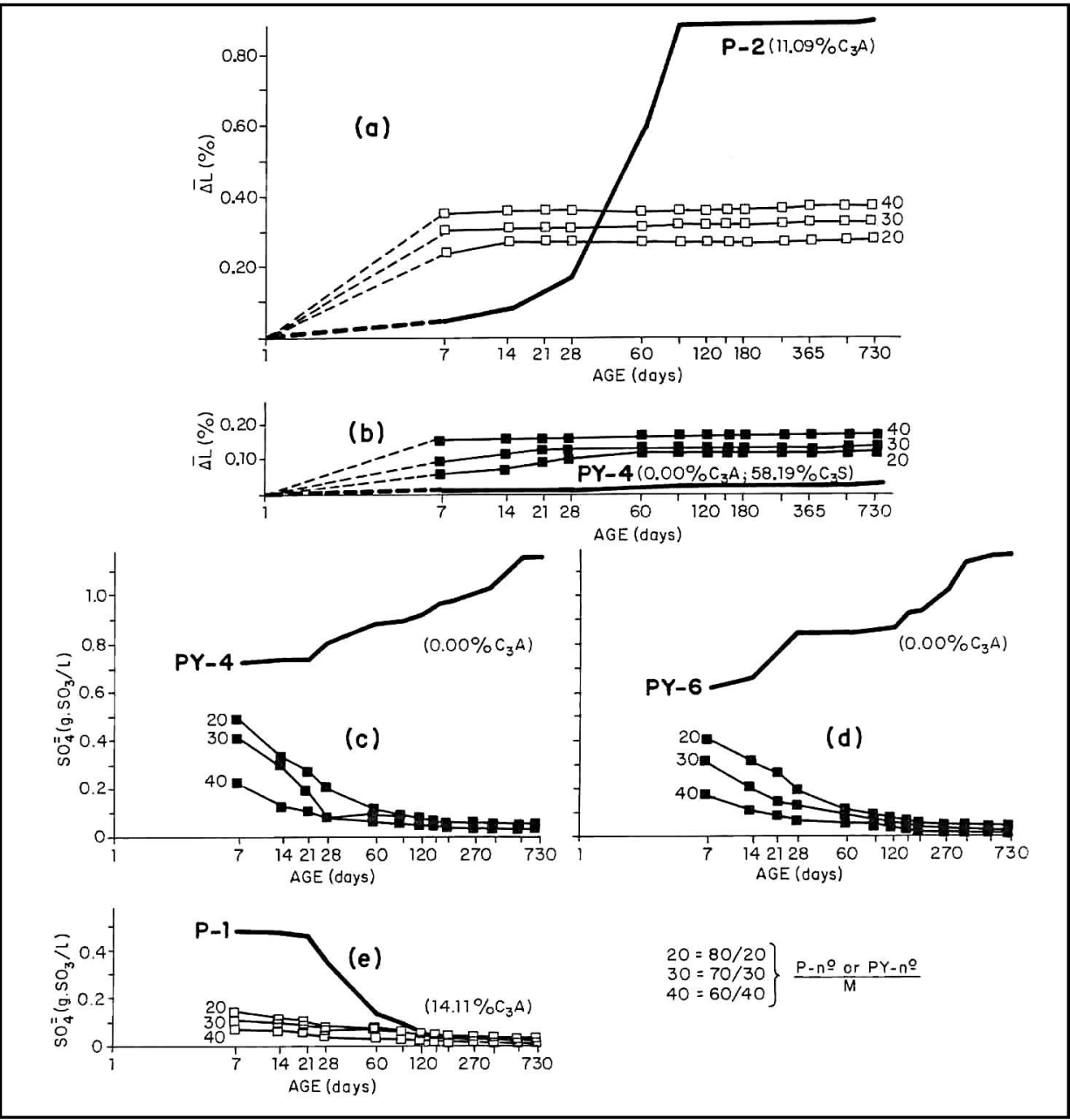

Figura 2. Probetas ASTM C 452-68 de las familias de PUZC [P-1/MK], [P-2/MK], [PY-4/MK] y $[\mathrm{PY}-6 / \mathrm{MK}]$. Parámetros: $\Delta \mathrm{L}(\%)$ y $\left\{\mathrm{SO}_{4}{ }^{2-}\right\} \mathrm{lcp}$. Figure 2. ASTM C 452-68 specimens of POZC families [P-1/MK], [P-2/MK], [PY-4/MK] and [PY-6/MK]. Parameters: $\Delta L(\%)$ and $\left\{\mathrm{SO}_{4}^{2-}\right\} \mathrm{CW}$. 
parámetros $\Delta \mathrm{L}(\%), \mathrm{Vcl}$ y $\left\{\mathrm{SO}_{4}{ }^{2-}\right\}_{\mathrm{lcp}}$ se determinaron a las edades de $1,7,28,60,90$ días y posteriores hasta dos años. De ellos, $\Delta \mathrm{L}(\%)$ y $\left\{\mathrm{SO}_{4}{ }^{2-}\right\}_{\mathrm{ICp}}$ fueron parámetros directos, mientras que la $\mathrm{Vcl}$ fue indirecto o relativo, habiéndose calculado sin más que dividir el $\Delta \mathrm{L}(\%)$ entre dos edades sucesivas por el número de días transcurrido entre ambas.
In this study, length was measured up to 2 years, and the Length Growth Rate, $\mathrm{Vcl}(=\Delta L(\%) /$ day) and $\left\{\mathrm{SO}_{4}{ }^{2-\}_{c w}}\right.$. However, the indirect or relative parameter used, Length Growth Rate, Vcl $(=\Delta L(\%) /$ day), was calculated by dividing the $\boldsymbol{\Delta L}(\%)$ by the relevant age (Figures $3(a, b, c, d)$ ).

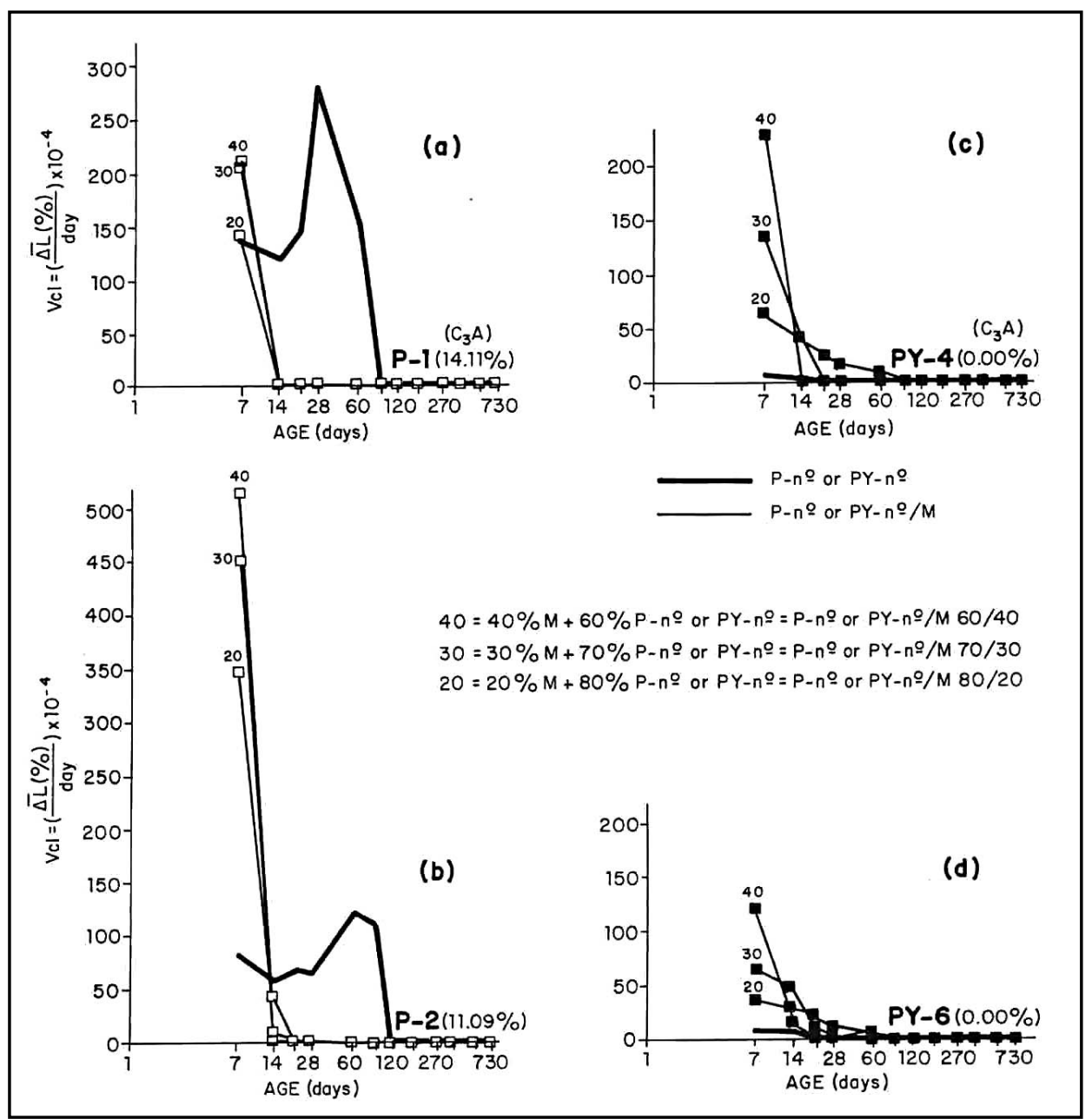

Figura 3. Probetas ASTM C 452-68 de las familias de PUZC [P-1/MK], [P-2/MK], [PY-4/MK] y [PY-6/MK]. Parámetro: Velocidad de crecimiento longitudinal $=\mathrm{Vcl}=(\Delta \mathrm{L}(\%) /$ day $)$.

Figure 3. ASTM C 452-68 specimens of POZC families [P-1/MK], $[P-2 / M K],[P Y-4 / M K]$ and $[P Y-6 / M K]$. Parameter: Length Growth Rate $=\mathrm{VCl}=(\Delta L(\%) /$ day $)$.

Tabla 4 / Table 4

Diferencias entre los contenidos de sulfatos teóricos y reales del agua de conservación de las probetas [P-1/M] a la edad de 7 días. Differences between theoretical and real sulphate contents in the curing water of $[P-1 / M]$ specimens at 7 days-age.

\begin{tabular}{|c|c|c|}
\hline Familia PUZC / POZC / family: [P-1/MK] & 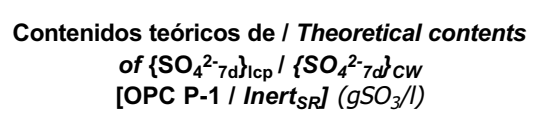 & 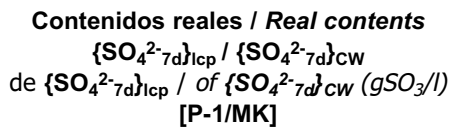 \\
\hline$[80 / 20]$ & $\frac{0.4710}{0.80}=0.58875-0.1021$ & $=0.48665$ fixed with $20 \%$ of MK \\
\hline$[70 / 30]$ & $\frac{0.4710}{0.70}=0.67290-0.0875$ & $=0.58540$ fixed with $30 \%$ of MK \\
\hline$[60 / 40]$ & $\frac{0.4710}{0.60}=0.78500-0.0711$ & $=0.71390$ fixed with $40 \%$ of MK \\
\hline
\end{tabular}


La relación del volumen de las probetas (de los nuevos morteros: probetas de $1^{\prime \prime} \times 11^{\prime \prime} \times 11^{1 / 4 " ;}$ de hormigón: $10 \times 10 \times 50 \mathrm{~cm}, \varnothing 15 \times 18 \mathrm{~cm}$ y $\varnothing 7,5 \times 15 \mathrm{~cm}$ ) al volumen de su agua de conservación, fue siempre el mismo -no mayor que $1 / 5$ - Para las probetas de 1 " $\times 11^{\prime \prime} \times 11^{1 / 4} 4^{\prime \prime}$ se utilizó agua destilada, pero para el resto de probetas no se utilizó agua destilada sino agua potable filtrada.

En la cuarta etapa, se le determinaron también a cada mortero tipo ASTM C 452-68 y edad del ensayo referida, el contenido de sulfatos $\left(\left\{\mathrm{SO}_{4}{ }^{2-}\right\}_{\mathrm{Ipp}}\right)$ en las aguas de almacenamiento y conservación (aguas de curado) de sus probetas de 1"x1"x10" (Figuras $2(c, d, e)$ ), utilizando para ello disolución de ácido clorhídrico $(\mathrm{ClH})$ y cloruro de bario $\left(\mathrm{BaCl}_{2}\right)$ para precipitarlos como sulfato de bario $\left(\mathrm{BaSO}_{4}\right)$ (Tabla 4). Otras determinaciones complementarias fueron análisis químico de los materiales cementíceos empleados y propiedades químicas y físicas específicas de algunos cementos ensayados (Tabla 1).

Finalmente, con los CP y sus PUZC con MK más adecuados, conteniendo 7,0\% ० 21,0\% $\mathrm{SO}_{3}$, se fabricaron hormigones (Tabla 5) para demostrar la formación de ESE, con los que se prepararon tres tipos diferentes de probetas:

1. probetas de $10 \times 10 \times 50 \mathrm{~cm}$, para determinarles su $\Delta \mathrm{L}(\%)$ en función del tiempo (Figura 4),

2. probetas de $\varnothing 7,5 \mathrm{~cm} \times 15 \mathrm{~cm}$ y $\varnothing 15 \mathrm{~cm} \times 30 \mathrm{~cm}$, para determinarles su resistencia mecánica a compresión, RMC, (48) y a tracción indirecta, RMTI, o "ensayo brasileño" (48) a 7, 28 y 90 días (Tablas 5 y 6), y

3. probetas de $\varnothing 15 \mathrm{~cm} \times 18 \mathrm{~cm}$ (48), para demostrar el ESE mediante el ensayo no destructivo de emisión acústica, EA, y más concretamente la energía de ultrasonido $(49,50)$ (Figura 5).
The ratio of the curing water volume to the volume of the specimens (new mortas: 1" $x 1$ " $x 10$ "; concretes: $10 \times 10 \times 50 \mathrm{~cm}, \varnothing 15 \times 18 \mathrm{~cm}$ and $\varnothing 7.5 \times 15 \mathrm{~cm}$ ) was always the same - not over 5 to 1 -. For 1 " $x 1^{\prime \prime} x 10^{\prime \prime}$ specimens, distilled water was used, but for the rest of specimens was not, but filtered potable water.

In the fourth step, the sulfate content of the storage water or curing water $\left(\left\{\mathrm{SO}_{4}{ }^{2-}\right\}_{\mathrm{cw}}\right)$ for the $1^{\prime \prime} x 1^{\prime \prime} x 10^{\prime \prime}$ specimens was also determined for each mortar and age tested (Figures $2(\mathrm{c}, \mathrm{d}, \mathrm{e})$ ), using hydrochloric acid $(\mathrm{ClH})$ and barium chloride $\left(\mathrm{BaCl}_{2}\right)$ to precipitate barium sulfate (Table 4). Other complementary determinations were chemical analysis of the cementing materials used and specific chemical and physical properties of some cements tested (Table 1).

Finally, with PC and their POZC with MK more adequate, three types of concrete specimens were also made to prove ESE, containing $7.0 \%$ or $21.0 \% \mathrm{SO}_{3}$. See dosage and results in Tables 5 and 6, respectively:

1. $10 \times 10 \times 50-\mathrm{cm}$ prisms, used to determine $\Delta L(\%)$ over time (Figure 4),

2. $\varnothing 15 \times 18-\mathrm{cm}$ and $\varnothing 7.5 \times 15-\mathrm{cm}$ cylinders, used to determine compressive strength (CS) (48) and indirect tensile strength (ITS) ("brazilian test") (48) at 7, 28, and 90 days (Table 6), and

3. Ø15x18-cm cylinders (48), used to find ESE via acoustic emission (AE) test, by ultrasound energy parameter (new non-destructive test) (49, 50) (Figure 5).

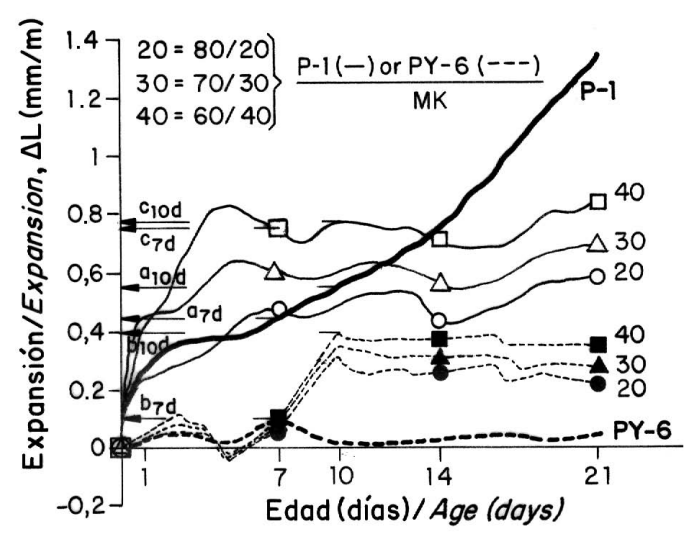

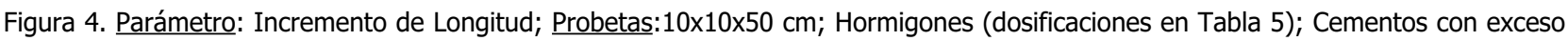
de $\left(7,0 \% \mathrm{SO}_{3}\right): 1$ OPC = P-1, 1 SRPC $=$ PY- 6 , y sus PUZC con MK; ESE entre ett-rf y ett-If: El valor real de $\Delta \mathrm{L}_{7 \mathrm{~d}}(\%)$ es $=\mathrm{C}_{7}, \mathrm{y}$ ha sido 2,64 veces mayor que su valor teórico $=\left[\left(60 \% \times a_{7 d}\right)+b_{7 d}\right]$ y el valor real de $\Delta \mathrm{L}_{10 \mathrm{~d}}(\%)$ es $=c_{10 d}$, $y$ ha sido 1,08 veces mayor que su valor teórico $=\left[\left(60 \% \times a_{10 d}\right)+b_{10 d}\right]$.

Figure 4. Parameter: Increase in Length; Specimens: 10x10x50 cm; Concretes (dosages are in Table 5); Cements with gypsum excess (7.0\% SO $\left.\mathrm{SO}_{3}\right): 1 \mathrm{OPC}=P-1,1 \mathrm{SRPC}=P Y-6$, and their POZC with MK; ESE between ett-rf and ett-If: Real $\Delta L_{7 d}(\%)$ value is $=c_{7}$, which has been 2.64-fold higher than its theoretical value $=\left[\left(60 \% x a_{7 d}\right)+b_{7 d}\right]$, and Real $\Delta L_{10 d}(\%)$ value is $=c_{10 d}$, which has been 1.08-fold higher than its theoretical value $=\left[\left(60 \% \times a_{10 d}\right)+b_{10 d}\right]$. 
Tabla 5 / Table 5

Hormigones: dosificación $\left(\mathrm{Kg} / \mathrm{m}^{3}\right)$.

Concretes: Dosage $\left(\mathrm{Kg} / \mathrm{m}^{3}\right)$.

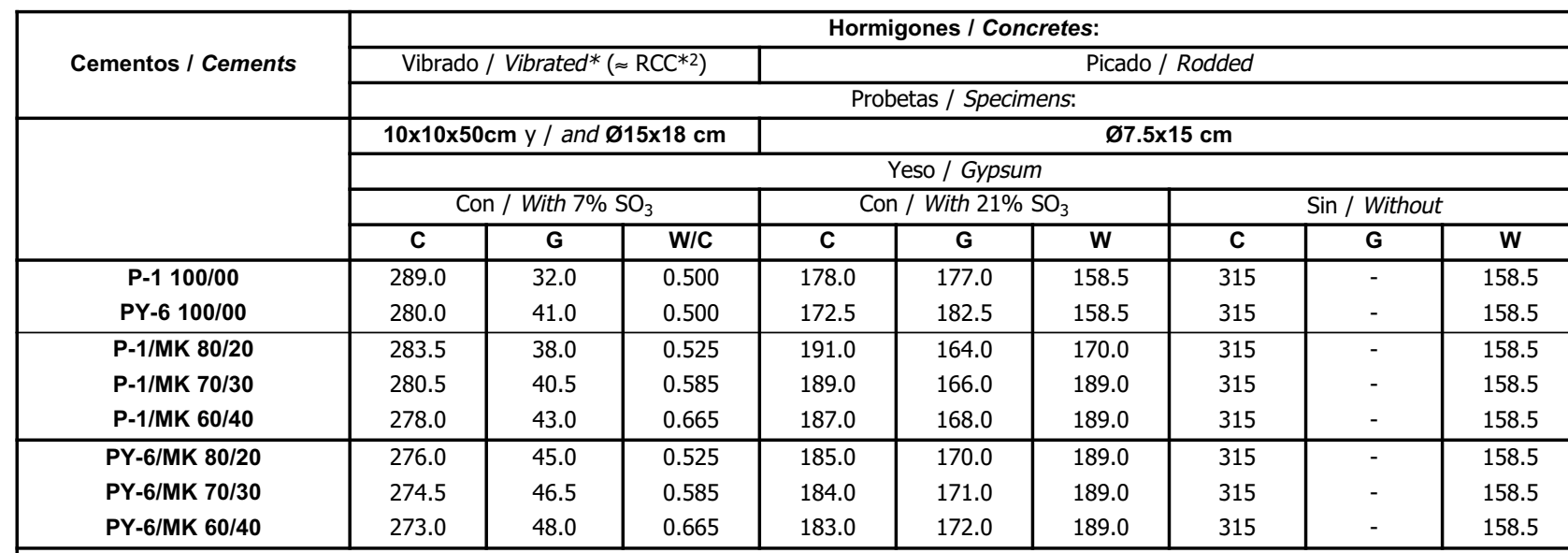

* Vibrado / Vibrated: Mediante una mesa vibro-compactadora / By means of the Vibro-compacting table; *2 Hormigón Compactado con Rodillo / Rolling Compacted Concrete $(=R C C)$

\begin{tabular}{|c|c|c|}
\hline \multicolumn{3}{|c|}{ Agregados / Aggregates*3 (calizo de machaqueo) / (crushing limestone) } \\
\hline - Grueso /Coarse & 643 & 654 \\
$10-20 \mathrm{~mm}$ & 436 & 436 \\
$5-10 \mathrm{~mm}$ & 1012 & 1017 \\
\hline - Fino / Fine & *3 Adsorción del árido grueso y fino / Absorption of coarse and fine aggregate $\approx 1.21 \% ;$ \\
\hline \multicolumn{3}{|c|}{ C = Cemento / Cement; G = Yeso / Gypsum; W = Agua / Water } \\
\hline \multicolumn{3}{|c|}{ Hormigones frescos / Fresh Concretes } \\
\hline \begin{tabular}{c|c|c} 
Peso específico / \\
Unit Weight
\end{tabular} & $2410 \mathrm{Kg} / \mathrm{m}^{3}$ & $2250 \mathrm{Kg} / \mathrm{m}^{3}$ \\
Asentamiento / Slump & $0.0 \mathrm{~cm}$ & $6.0 \mathrm{~cm}$ \\
Vibrado / Vibrated & $8 \approx \mathrm{RCC}$ & Picado / Rodded \\
\hline
\end{tabular}

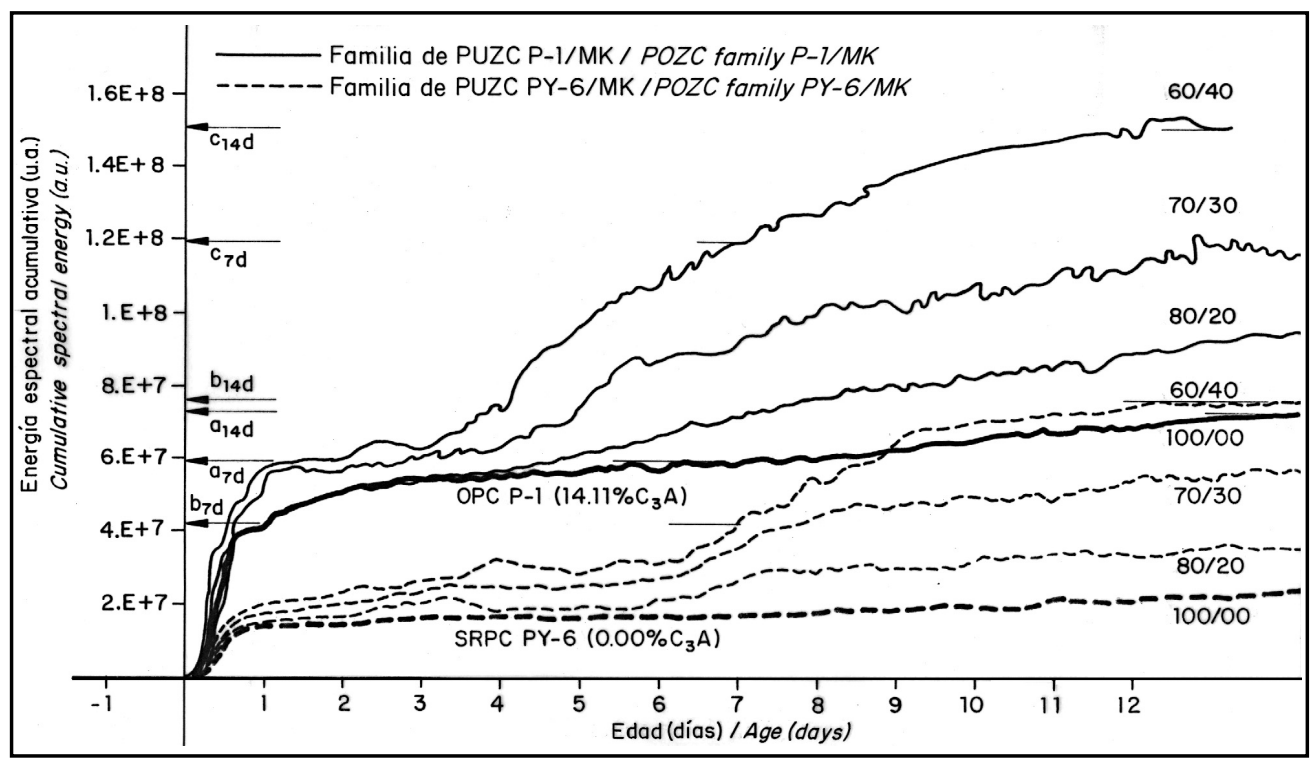

Figura 5.- Parámetro: Espectro de Energía de Ultrasonido (EU). Cantidad de energía recogida; Probetas: Ø15x18 cm; Hormigones, cuyas dosificaciones se encuentran en la Tabla 5; Cementos con yeso $\left(=7,0 \% \mathrm{SO}_{3}\right): 1 \mathrm{OPC}=\mathrm{P}-1,1 \mathrm{SRPC}=\mathrm{PY}-6$, y sus POZC con MK; ESE entre la ett-rf y la ett-If: El valor real de $\mathrm{EU}_{7 \mathrm{~d}}$ es $=c_{7}$, y ha sido 1,54 veces mayor que su valor teórico $=\left[\left(60 \% \mathrm{x} \mathrm{a}_{7 \mathrm{~d}}\right)+\mathrm{b}_{7 \mathrm{~d}}\right]$, y El valor real de EU es $=c_{10 d}$ y ha sido 1,25 veces mayor que su valor teórico $=\left[\left(60 \% \times a_{10 d}\right)+b_{10 d}\right]$.

Figure 5. Parameter: Cumulative energy power spectrum or ultrasound energy y(UE); Specimens: $\varnothing 15 \times 18 \mathrm{~cm}$; Concretes whose dosages are in Table 5; Cements with gypsum excess $\left(=7.0 \% \mathrm{SO}_{3}\right): 1 \mathrm{OPC}=P-1,1 \mathrm{SRPC}=\mathrm{PY}-6$, and their POZC with MK; ESE between ett-rf and ett-If: Real UE value is $=c_{7}$, which has been 1.34-fold higher than its theoretical value $=\left[\left(60 \% \times a_{7 d}\right)+b_{7 d}\right]$, and Real UE value is $=c_{10 d}$, which has been 1.25-fold higher than its theoretical value $=\left[\left(60 \% \times a_{10 d}\right)+b_{10 d}\right]$. 
Tabla 6 / Table 6

Resistencias mecánicas de los diferentes hormigones. Mechanical strengths of the different concretes.

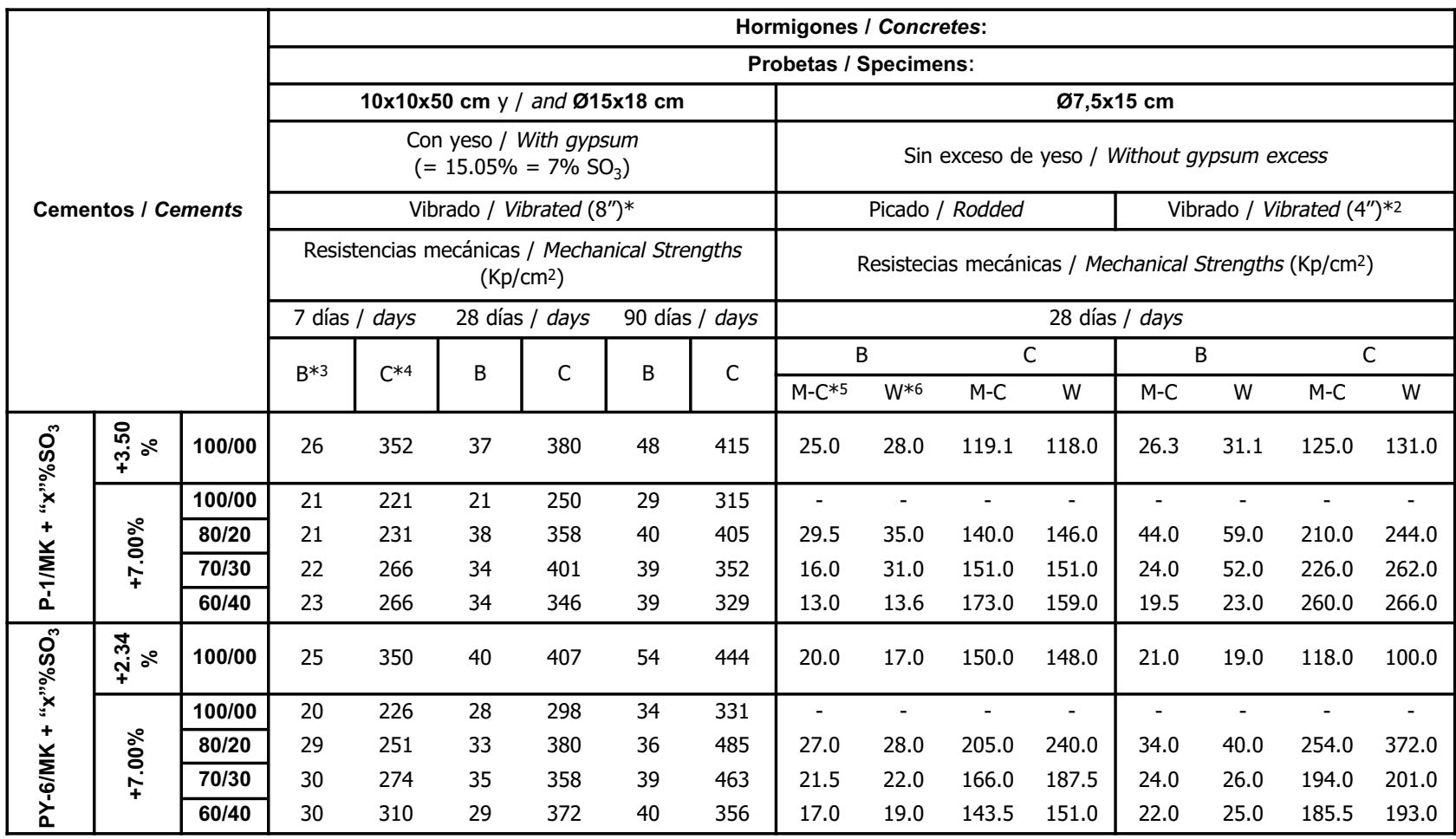

* = Consistencia seca / Dry Consistence.

*2 = Consistencia plástica / Plastic Consistence.

*3 = Tracción indirecta / Indirect Traction Strength (ensayo "brasileño" / "brazilian" test).

*4 = Resistencia a compresión / Compresive Strength.

*5 = Cámara húmeda / Moist-Cured.

$* 6$ = En agua / In water.

\section{DISCUSIÓN}

Las técnicas analíticas y los parámetros químicos, físicos y mecánico-resistentes que se han considerado necesarios para poder demostrar la existencia de ESE entre la ett-rf (de origen $\mathrm{Al}_{2} \mathrm{O}_{3}{ }^{\mathrm{r}-}$ presente en el $\mathrm{MK}$ ), y la ett-If (del $\mathrm{C}_{3} \mathrm{~A}$ presente en $\mathrm{CPO}$ ) cuando precipitan conjuntamente (co-precipitación) en un mismo medio selenitoso común, han sido los siguientes:

- Con los morteros de cemento tipo ASTM C 452-68. Mediante los siguientes parámetros: $\Delta \mathrm{L}(\%), \mathrm{Vcl}$, resistencia mecánica a compresión, $\mathrm{RMC}$, resistencia mecánica a flexotracción, RMF, y contenido de sulfatos en las aguas de conservación de las probetas de $1^{\prime \prime} x 1^{\prime \prime} x 11^{1 / 4 "},\left\{\mathrm{SO}_{4}{ }^{2-}\right\}_{\text {Icp, }} \mathrm{y}$

- Con los hormigones de cemento. Mediante los siguientes parámetros: Resistencia mecánicas a compresión (RMC) y tracción indirecta (RMTI) o ensayo "brasileño"), $\Delta \mathrm{L}(\mathrm{mm} / \mathrm{m})$ y $\mathrm{EA}$, su parámetro energía de ultrasonido, EU, especialmente.

\section{DISCUSSION}

The analytical techniques, test methods and physical, chemical and mechanical strength parameters considered necessary to prove the existence of ESE between ett-rf (from the $\mathrm{Al}_{2} \mathrm{O}_{3}{ }^{r-}$ present in M pozzolan), and ett-If (from the $C_{3} A$ present in $O P C$ ) when precipitating jointly (co-precipitation) in the same plaster-bearing solution, were as follows,

- For cement mortars.- ASTM C 452-68 test, by means of the following parameters: $\Delta L(\%), \mathbf{V c l}(=\Delta L$ $(\%) / d a y)$, MS (CS and FS), and $\left\{\mathrm{SO}_{4}{ }^{2-\}_{c w}}\right.$ and

- For cement concretes.- By means of the following parameters: $\boldsymbol{M S}$ (CS and ITS), $\boldsymbol{\Delta L}(\mathrm{mm} / \mathrm{m})$ and acoustic emission (AE) test by its ultrasound energy parameter especially. 
Finalmente, se compararán en cada caso los PUZC más adecuados, según el parámetro que se ha considerado más apropiado para probar la existencia de ESE, o simplemente, Efecto Sinérgico, ES, en el caso del parámetro $\left\{\mathrm{SO}_{4}{ }^{2-}\right\}_{\text {Icp. }}$.

\subsection{Con los morteros de cemento: Ensayo ASTM C 452-68 (15) (con 7,0\% $\mathrm{SO}_{3}$ )}

\subsubsection{Mediante los parámetros $\Delta \mathrm{L}(\%)$ y Vcl}

\subsubsection{Valores de $\Delta \mathrm{L}(\%)$ de las probetas ASTM C 452-68}

El caso más favorable para demostrarlo se encuentra comparando las familias de PUZC P-2/MK y PY-4/MK (Figuras 2(a)(b)). Así se tiene que, el $\Delta \mathrm{L}_{7 \mathrm{~d}}(\%)$ de las probetas del CPO puro [PY-4 100/00] es $=0,005 \%$, y el $80 \%$ de este valor es $=0,004 \%$, el $70 \%=0,0035 \%$, y el $60 \%=0,0030 \%$. Además,

(a). La diferencia entre cada valor parcial de $\Delta \mathrm{L}_{7 \mathrm{~d}}(\%)$ y el valor de $\Delta \mathrm{L}_{7 \mathrm{~d}}(\%)$ de las respectivas probetas [PY-4/MK] [80/20], [70/30] y [60/40] tiene que ser debido al 20\%, $30 \%$ y $40 \%$ de $\mathrm{MK}+7,0 \% \mathrm{SO}_{3}$, respectivamente, es decir,

- $0,044 \%-0,0040 \%=0,0400 \%$ de $\Delta \mathrm{L}_{7 \mathrm{~d}}=\left(\mathrm{a}_{1}\right)$, el cual sería debido solamente, al $20 \%$ del MK de la mezcla,

- $0,095 \%-0,0035 \%=0,0915 \%$ de $\Delta \mathrm{L}_{7 \mathrm{~d}}=\left(\mathrm{b}_{1}\right)$, el cual sería debido solamente, al $30 \%$ del MK de la mezcla, y

- $0,160 \%-0,0030 \%=0,1570 \%$ de $\Delta \mathrm{L}_{7 \mathrm{~d}}=\left(\mathrm{c}_{1}\right)$, el cual sería debido solamente, al $40 \%$ del MK de la mezcla.

(b). Los valores $80 \%$, $70 \%$ y $60 \% \times \Delta \mathrm{L}_{7 \mathrm{~d}}(\%)$ de las probetas [P-2 100/00] serían 80\%x0,058\%, 70\%x0,058\% y $60 \% \times 0,058 \%$, es decir, $=0,0464 \%\left(=a_{2}\right), 0,0406 \%$ $\left(=b_{2}\right)$, y $0,0348 \%\left(=c_{2}\right)$, respectivamente.

Finalmente, sumando ahora de manera ordenada las dos últimas tríadas de cálculos numéricos realizados, se deberán obtener los valores teóricos de $\Delta \mathrm{L}_{7 \mathrm{~d}}(\%)$ que los PUZC P-2/MK 80/20, 70/30 y 60/40, respectivamente, deberían haber originado, es decir,

- $\left(\mathrm{a}_{1}\right)+\left(\mathrm{a}_{2}\right)=0,0400 \%+0,0460 \%=0,0860 \%$,

- $\left(b_{1}\right)+\left(b_{2}\right)=0,0915 \%+0,0406 \%=0,1321 \%, y$

- $\left(c_{1}\right)+\left(c_{2}\right)=0,1570 \%+0,0340 \%=0,1910 \%$.

Sin embargo, tales PUZC han originado unos valores reales de $\Delta \mathrm{L}_{7 \mathrm{~d}}(\%)$ de $0,242 \%, 0,310 \%$ y $0,360 \%$, respectivamente, es decir, 2,81, 2,35 y 1,88 veces mayores, que los correspondientes teóricos. Lo que es una señal inequívoca del notable ESE originado en este caso, pudiéndose
Finally and in order to prove the existence of ESE or simply ES, the most adequate POZC will be compared according to the more appropriate parameter considered for each case, i.e., the sulfate contents chemical parameter, will be determined in their respective curing water, $\left\{\mathrm{SO}_{4}{ }^{2-}\right\}_{\mathrm{cw}}$.

\subsection{Cement mortars type: ASTM C 452-68 test (15) (with $7.0 \% \mathrm{SO}_{3}$ )}

\subsubsection{By means of the $\Delta L(\%)$ y Vcl parameters}

\subsubsection{1. $\Delta L(\%)$ values for the ASTM C 452-68 specimens}

The most suitable method to prove the existence of ESE is by comparing POZC families $P-2 / M$ and $P Y-4 / M$ (Figures 2(a)(b)). Thus, $\Delta L_{7 d}(\%)$ for the plain $[100 / 00$ PY-4] specimens $=0.005 \%$, and $80 \%$ of this value $=$ $0.004 \%, 70 \%=0.0035 \%$, and $60 \%=0.0030 \%$. Moreover,

(a). The difference between each partial $\Delta L_{7 d}(\%)$ value and the $\Delta L_{7 d}(\%)$ value for the respective [80/20], [70/30] and [60/40] [PY-4/M] specimens must be due to the $20 \%$, $30 \%$ and $40 \%$ of $\mathrm{MK}+7.0 \% \mathrm{SO}_{3}$, respectively, i.e.

- $0.044 \%-0.0040 \%=0.0040 \%$ of $\Delta L_{7 d}=\left(a_{1}\right)$, which would be totally due to the $20 \%$ of MK in the mix,

- $0.095 \%-0.0035 \%=0.0915 \%$ of $\Delta L_{7 d}=\left(\mathbf{b}_{1}\right)$, which would be totally due to the $30 \%$ of MK in the mix, and

- $0.160 \%-0.0030 \%=0.1570 \%$ of $\Delta L_{7 d}=\left(c_{1}\right)$, which would be totally due to the $40 \%$ of MK in the mix.

(b). The $80 \%, 70 \%$ and $60 \% x \Delta L_{7 d}(\%)$ values for the [100/00 P-2] specimens would be $80 \%, 70 \%$ and $60 \% \times 0.058 \%$, or $=0.0464 \%\left(=a_{2}\right), 0.0406 \%\left(=b_{2}\right)$, and $0.0348 \%\left(=c_{2}\right)$, respectively.

Finally, summing the foregoing sets of numerical results yields the respective theoretical $\Delta L_{7 d}(\%)$ values for the [80/20], [70/30] and [60/40] [P-2/M] specimens, namely:

- $\left(a_{1}\right)+\left(a_{2}\right)=0.04000 \%+0.0464 \%=0.0864 \%$,

- $\quad\left(b_{1}\right)+\left(b_{2}\right)=0.09150 \%+0.0406 \%=0.1321 \%$, and

- $\left(c_{1}\right)+\left(c_{2}\right)=0.15700 \%+0.0340 \%=0.1910 \%$.

However, the real $\Delta L_{7 d}(\%)$ values for the [80/20], [70/30] and [60/40] [P-2/M] specimens were 0.242\%, $0.310 \%$ y $0.360 \%$, respectively, i.e. substantially higher than their respective theoretical $\Delta \mathbf{L}_{\mathbf{7 d}}(\%)$ values. This kind of behavior is a well-defined sign of the notable 
calificar por el contrario, de bastante menor e incluso escaso para la familia de PUZC P-1/MK.

En definitiva, los valores reales de $\Delta \mathrm{L}_{7 \mathrm{~d}}(\%)$ de las probetas [P-2/MK] [80/20], [70/30] y [60/40] resultaron ser $2,8,2,4$ y 1,9 veces superiores que sus correspondientes valores teóricos, y como resultado, se ha producido un notable ESE de resultado adverso en este caso. Continúese más adelante en el apartado 4.3. Consecuencias técnicas.

\subsubsection{Valores de Vcl de las probetas ASTM C 452-68}

El caso más favorable para la demostrarlo se encuentra entre las familias de PUZC P-1/MK, P-2/MK y PY-6/MK (Figuras 3 ( $a, b, c, d)$ ). Así, en la PY-6/M se tiene que,

- el valor de $\mathrm{VCl}_{7 \mathrm{~d}}$ del $20 \%$ de MK en las probetas [PY$6 / \mathrm{MK}$ 80/20] debe ser $=0,00357 \Delta \mathrm{L}(\%) /$ día $(0,8 \times 0,00057 \Delta \mathrm{L}(\%) /$ día $)=0,00311 \Delta \mathrm{L}(\%) /$ día,

- el valor de $\mathrm{VCl}_{7 \mathrm{~d}}$ del $30 \%$ de MK en las probetas [PY$6 / \mathrm{MK}$ 70/30] debe ser $=0,00657 \Delta \mathrm{L}(\%) /$ día $(0,7 \times 0,00057 \Delta \mathrm{L}(\%) /$ día $)=0,00617 \Delta \mathrm{L}(\%) /$ día

- el valor de $\mathrm{VCl}_{7 \mathrm{~d}}$ del $40 \%$ de MK en las probetas [PY6/MK 60/40] debe ser $=0,01200 \Delta \mathrm{L}(\%) /$ día $(0,6 \times 0,00057 \Delta \mathrm{L}(\%) / d i ́ a)=0,01166 \Delta \mathrm{L}(\%) / d i ́ a$,

- Los valores de $\mathrm{Vcl}_{7 \mathrm{~d}}$ del $80 \%, 70 \%$ y $60 \%$ de las probetas [P-1] deben ser $=0,01343 \Delta \mathrm{L}(\%) /$ díax0,8, $\mathrm{x} 0,7$, y $\mathrm{x} 0,6=0,010744 \Delta \mathrm{L}(\%) /$ día, 0,0094 $\Delta \mathrm{L}(\%) / d i ́ a$, y $0,008058 \% \Delta \mathrm{L}(\%) /$ día, respectivamente.

- Los valores teóricos de $\mathrm{Vcl}_{7 \mathrm{~d}}$ de las probetas [P$1 / \mathrm{MK}][80 / 20],[70 / 30]$ y $[60 / 40]$ deben ser $=$ $0,00311+0,01075=0,01386 \Delta \mathrm{L}(\%) /$ día, $0,00617+$ $0,0094=0,01557 \Delta \mathrm{L}(\%) /$ día y $0,01166+0,00806=$ $0,01972 \Delta \mathrm{L}(\%) /$ día, respectivamente, mientras sus respectivos valores reales fueron $0,01414 \Delta \mathrm{L}(\%) /$ día, $0,02014 \Delta \mathrm{L}(\%) /$ día y $0,02114 \Delta \mathrm{L}(\%) /$ día.

- Los valores de $\mathrm{VCl}_{7 \mathrm{~d}}$ del $80 \%, 70 \%$ y $60 \%$ [P-2] deben ser $=0,00829 \Delta \mathrm{L}(\%) /$ día $\times 0,8, \times 0,7$ y $\times 0,6=$ $0,006632 \Delta \mathrm{L}(\%) /$ día, $0,005803 \Delta \mathrm{L}(\%) /$ día $\quad \mathrm{y}$ $0,004974 \% \Delta \mathrm{L}(\%) /$ día, respectivamente.

- Los valores teóricos de $\mathrm{Vcl}_{7 d}$ de las probetas [P$2 / \mathrm{MK}][80 / 20],[70 / 30]$ y $[60 / 40]$ deben ser $=$ $0,00311+0,006632=0,00974 \Delta \mathrm{L}(\%) /$ día, 0,00617 $+0,005803=0,01197 \Delta \mathrm{L}(\%) /$ día y $0,01166+$ $0,004974=0,01663 \Delta \mathrm{L}(\%) / d i ́ a$, respectivamente, mientras sus respectivos valores reales fueron $0,03457 \Delta \mathrm{L}(\%) /$ día, $0,04486 \Delta \mathrm{L}(\%) /$ día y 0,05143 $\Delta \mathrm{L}(\%) /$ día.

Así y en función de este parámetro $\mathrm{Vcl}_{7 \mathrm{~d}}$, de las probetas tipo ASTM C 452-68 de estos PUZC, el ESE se determinaría mediante la diferencia, a igualdad de PUZC, entre los correspondientes valores teóricos y reales de $\mathrm{Vcl}_{7 d}$. Por tanto:
ESE originated in this case, but in contrast, and according to the POZC $P-1 / M K$ family results, it is all the more insignificant.

In short, by the age of 7 days, then, considerable ESE of adverse result in this case, had taken place in the ASTM C 452-68 specimens, inasmuch as the real $\Delta L_{7 d}(\%)$ values for [80/20], [70/30] and [60/40] [P-2/MK] specimens were 2.8-, 2.4- and 1.9-fold higher than their respective theoretical $\Delta L_{7 d}(\%)$ values. See points 1 and 2 in 4.3. Technical Consequences, later on.

\subsubsection{Vcl values for the ASTM C 452-68 specimens}

The most suitable method to prove the existence of ESE is by comparing POZC families $P-1 / M K, P-2 / M K$ and $P Y$ $6 /$ MK (Figures 3 ( $a, b, c, d)$ ). Thus,

- $\mathrm{VCl}_{7 d}$ for the $20 \%$ of MK in the mix for the [80/20 PY$6 / \mathrm{M}]$ specimens must be $=0.00357 \Delta L(\%) /$ day $(0.8 \times 0.00057 \Delta L(\%) /$ day $)=0.00311 \Delta L(\%) / d a y$,

- $\mathrm{VCl}_{7 d}$ for the $30 \%$ of MK in the mix for the [70/30 PY$6 / M K]$ specimens must be $=0.00657 \Delta L(\%) /$ day $(0.7 \times 0.00057 \Delta L(\%) / d a y)=0.00617 \Delta L(\%) / d a y$,

- $\mathrm{VCl}_{7 d}$ for the $40 \%$ of MK in the mix for the [60/40 PY$6 / M K]$ specimens must be $=0.01200 \Delta L(\%) /$ day $(0.6 \times 0.00057 \Delta L(\%) / d a y)=0.01166 \Delta L(\%) / d a y$,

- $\quad \mathrm{VCl}_{7 d}$ for the $80 \%, 70 \%$ and $60 \%$ of [P-1] specimens must be $=0.01343 \Delta L(\%) /$ day $x 0.8, \times 0.7$, and $\times 0.6$ $=0.010744 \Delta L(\%) / d a y, 0.0094 \Delta L(\%) /$ day, and $0.008058 \% \Delta L(\%) /$ day, respectively.

- The theoretical $\mathrm{VCl}_{7 d}$ values for the [80/20], [70/30] and [60/40] [P-1/MK] specimens must be $=0.00311$ $+0.01075=0.01386 \Delta L(\%) /$ day, $0.00617+0.0094$ $=0.01557 \Delta L(\%) /$ day and $0.01166+0.00806=$ $0.01972 \Delta L(\%) /$ day, respectively, whilst their respective real $\mathrm{VCl}_{7 d}$ values were $0.01414 \Delta \mathrm{L}(\%) /$ day, $0.02014 \Delta L(\%) /$ day and $0.02114 \Delta L(\%) /$ day.

- $\mathrm{VCl}_{7 d}$ values for the $80 \%, 70 \%$ and $60 \%$ of [P-2] specimens must be $=0.00829 \Delta L(\%) /$ day $\times 0.8$, $x 0.7$, and $x 0.6=0.006632 \Delta L(\%) / d a y, 0.005803$ $\Delta L(\%) / d a y$, and $0.004974 \Delta L(\%) /$ day, respectively.

- The theoretical $\mathrm{Vcl}_{7 d}$ values for [80/20], [70/30] and [60/40] [P-2/MK] specimens must be $=0.00311+$ $0.006632=0.00974 \Delta L(\%) / d a y, 0.00617+0.005803$ $=0.01197 \Delta \mathrm{L}(\%) /$ day and $0.01166+0.004974=$ $0.01663 \Delta L(\%) /$ day, respectively, whilst their respective real $\mathrm{VCl}_{7 d}$ values were 0.03457 $\Delta L(\%) /$ day, $0.04486 \Delta L(\%) /$ day and 0.05143 $\Delta L(\%) / d a y$.

ESE in terms of the Length Growth Rate, Vcl, of these ASTM C 452-68 specimens would be measured as the difference -for the same POZC- between the respective real and theoretical $\mathbf{V c l}_{7 d}$ values. Hence: 
- Para las probetas [P-1/MK $80 / 20]$, la diferencia fue $0,00056 \Delta \mathrm{L}(\%) /$ día,

- Para las probetas [P-1/MK 70/30], fue 0,000457 $\Delta \mathrm{L}(\%) /$ día,

- Para las probetas [P-1/MK 60/40], fue 0,001424 $\Delta \mathrm{L}(\%) /$ día,

- Para las probetas [P-2/MK 80/20], fue 0,024830 $\Delta \mathrm{L}(\%) / d i ́ a$,

- Para las probetas [P-2/MK 70/30], fue 0,032890 $\Delta \mathrm{L}(\%) / d i ́ a, y$

- Para las probetas [P-2/MK 60/40], fue 0,034800 $\Delta \mathrm{L}(\%) / d i ́ a$.

En definitiva, los valores reales de $\mathrm{VCl}_{7 \mathrm{~d}}(\%)$ de las probetas $[\mathrm{P}-1 / \mathrm{MK}]$ y $[\mathrm{P}-2 / \mathrm{MK}][80 / 20],[70 / 30]$ y $[60 / 40]$ resultaron ser $1,02,1,29,1,07,3,54,3,75$ y 3,09 veces mayores que los teóricos, y como consecuencia, se ha producido un notable ESE de resultado adverso en este caso. Continúese más adelante en el apartado 4.3. Consecuencias técnicas.

4.1.1.3. Mediante el parámetro $\left\{\mathrm{SO}_{4}{ }^{2-}\right\}_{\mathrm{Icp}}$ del agua de conservación de las probetas ASTM C 452-68

Los resultados de este parámetro químico se encuentra en las Figuras 2(c)(e)(d) y en la Tabla 4, y para ello se van a considerar dos supuestos, a saber:

- $\quad 1^{\text {er }}$ Supuesto: caso de que los cementos PY-4 y PY-6 no tienen nada en absoluto de $\mathrm{C}_{3} \mathrm{~A}$, según los cálculos de Bogue, y por tanto, no deberían fijar $\mathrm{SO}_{3}$ del $7,0 \%$ inicial para originar ett-If.

- 20 Supuesto: caso de que los cementos PY-6 y PY-4 tienen muy poco $\mathrm{C}_{3} \mathrm{~A}\left(<1,0 \% \mathrm{C}_{3} \mathrm{~A}\right)$ pese a los cálculos de Bogue, y por tanto, fijarán muy poco $\mathrm{SO}_{3}$ del 7,0\% inicial para originar ett-If.

Desarrollo del razonamiento:

1er Supuesto: Si tal es el caso, la cantidad de $\left\{\mathrm{SO}_{4}{ }^{2-}\right\}$ Icp7d habría de ser exactamente igual tanto si el cemento Pórtland PY está solo, como si está mezclado con un

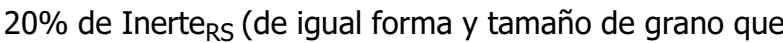
el $20 \%$ de cemento PY sustituido), es decir, la cantidad de $\left\{\mathrm{SO}_{4}{ }^{2-}\right\}_{4 l \mathrm{lcp} 7 \mathrm{~d}}$ del cemento PY-4 sólo ha sido $0,7210 \mathrm{~g}$ $\mathrm{SO}_{3} / \mathrm{l}$ y la cantidad de $\left\{\mathrm{SO}_{4}{ }^{2-}\right\}_{\text {Icp7d }}$ del cemento de mezcla PY-4/Inerte ${ }_{\mathrm{RS}} 80 / 20$ debe de ser también $0,7210 \mathrm{~g}$ $\mathrm{SO}_{3} / \mathrm{l}$; sin embargo ha sido $0,4895 \mathrm{~g} \mathrm{SO}_{3} / \mathrm{l}$ en el caso del MK, o sea, en el PUZC PY-4/MK 80/20, Luego dicho $20 \%$ de $M K$ ha sido la causa de que el $0,7210 \mathrm{~g} \mathrm{SO}_{3} / \mathrm{l}$ haya disminuido hasta $0,4072 \mathrm{~g} \mathrm{SO}_{3} /$ l. Por lo tanto, dicha fracción de $\mathrm{MK}(20 \%)$ habrá fijado 0,7210 - 0,4895 = $0,2315 \mathrm{~g} \mathrm{SO}_{3} /$.
- for the $[80 / 20 P-1 / M K]$ specimens, the difference was $0.00056 \Delta L(\%) / d a y$,

- for the [70/30 P-1/MK] specimens, 0.000457 $\Delta L(\%) / d a y$,

- for the [60/40 P-1/MK] specimens, 0.001424 $\Delta L(\%) / d a y$,

- for the [80/20 P-2/MK] specimens, 0.024830 $\Delta L(\%) / d a y$,

- for the [70/30 P-2/MK] specimens, 0.032890 $\Delta L(\%) / d a y$, and

- for the [60/40 P-2/MK] specimens, 0.034800 $\Delta L(\%) / d a y$.

In short, by the age of 7 days, then, substantial ESE of adverse result as well, had taken place in ASTM C 45268 specimens, inasmuch as the real $\mathrm{Vcl}_{7 d}(\%)$ values for [80/20], [70/30] and [60/40] [P-1/MK] and [P-2/MK] specimens were 1.02-, 1.29-, 1.07-, 3.54-, 3.75- and 3.09-fold higher than their respective theoretical $\mathrm{Vcl}_{7 d}$ (\%) values. See points 1 and 2 in 4.3. Technical Consequences, later on.

4.1.1.3. Sulfate content in the storage water of ASTM $452-68$ specimens $=\left\{\mathrm{SO}_{4}{ }^{2-}\right\}_{\mathrm{CW}}$.

Results of this chemical parameter are in Figures $2(c)(d)(e)$ and Table 4, and one of two assumptions is made in this comparison, namely:

- Assumption 1: $C_{3} A$ (\%) content is zero for both the PY-4 and PY-6 SRPC, as per Bogue calculation criteria, and therefore neither SRPC must react with the initial $7.0 \% \mathrm{SO}_{3}$ added to originate ett-If.

- Assumption 2: $C_{3} A(\%)$ content is $<1.0 \%$ for both the PY-4 and PY-6 SRPC, contrary to Bogue calculation criteria, and therefore both SRPC must react with the initial $7.0 \% \mathrm{SO}_{3}$ added to originate ettIf, albeit scantily.

Discussion of the reasoning followed:

Assumption 1: In this case, the amount of $\left\{\mathrm{SO}_{4}{ }^{2-\}_{c w 7 d}}\right.$ would have to be exactly the same both if plain SRPC PY is used or if it is blended with $20 \%$ of a sulfate-inert substance (in the same way and with the same grain size as the 20\% MK); in other words, since the amount of $\left\{\mathrm{SO}_{4}{ }^{2-}\right\}_{\text {cw7d }}$ in plain PY-4 was $0.7210 \mathrm{~g} \mathrm{SO}_{3} / \mathrm{l}$, the amount of $\left\{\mathrm{SO}_{4}{ }^{2-}\right\}_{\mathrm{cw7d}}$ in the $80 / 20$ blended $\mathrm{PY}$ 4/Intert $t_{S R}$ substance cement should also be $0.7210 \mathrm{~g}$ $\mathrm{SO}_{3} / \mathrm{l}$. However, the amount for blended cement with $20 \%$ MK, [80/20 PY-4/MK] POZC, was 0,4895. Therefore, the drop from 0.7210 to 0.4072 can be attributed to the presence of the $20 \% M K$ and it must consequently be deduced that the $20 \% \mathrm{MK}$ has reacted with $0.2315 \mathrm{~g}$ $\mathrm{SO}_{3} / \mathrm{l}=0.7210 \mathrm{~g} \mathrm{SO}_{3} / \mathrm{l}-0.4895 \mathrm{~g} \mathrm{SO}_{3} / \mathrm{l}$. 
Y del mismo modo, en el caso del cemento PY- 6 se tendrá: $0,6114-0,4008=0,2106 \mathrm{~g} \mathrm{SO}_{3} / \mathrm{l}$. Por lo que tomando ahora el valor medio de ambos casos posibles, se tendría que el $20 \%$ de MK fijaría ella sola 0,2622 $\mathrm{g} \mathrm{SO}_{3} / \mathrm{l}$.

Pues bien, razonando igualmente para el caso del cemento Pórtland matriz acompañante P-1 (14,11\% $C_{3} A$ ) solo en su PUZC $P-1 / M K ~ 80 / 20$, se tendría que:

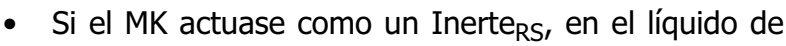
conservación de las probetas, habría habido 0,4710 / $0,8=0,58875 \mathrm{~g} \mathrm{SO}_{3} / \mathrm{l}\left(0,4710 \mathrm{~g} \mathrm{SO}_{3} / \mathrm{l}\right.$ es del CP P-1 solo a la edad de 7 días), y eso no ha ocurrido, pero:

- Si el MK no actuase como un Inerte $\mathrm{RS}$ y si en cambio como tal puzolana, habría dejado libre 0,58875 - 0,22105 = 0,3677 $\mathrm{g} \mathrm{SO}_{3} /$ l.iY eso no ha ocurrido tampoco! Ya que en la realidad ha quedado libre menor cantidad de $\left\{\mathrm{SO}_{4}{ }^{2-}\right\}_{\text {lcp7d }}$, o sea, $0,1021 \mathrm{~g} \mathrm{SO}_{3} / \mathrm{l}$ (contenido real) , es decir, tres veces menos, por haberse comportado el MK como una verdadera puzolana. Lo cual es tanto como decir que $20 \%$ de MK reaccionó tres veces más activamente con el contenido inicial de yeso $\left(15,05 \%=7,0 \% \mathrm{SO}_{3}\right)$ de su PUZC que si el MK hubiera sido un material "inerte". Luego esta importante actividad puzolánica del MK ha producido en esta última situación un Efecto Sinérgico, ES, entre la $\mathrm{Al}_{2} \mathrm{O}_{3}{ }^{\mathrm{r}-}$ de la fracción MK $(20 \%, 30 \%$ y $40 \%)$ de cada PUZC y el $\mathrm{C}_{3} \mathrm{~A}$ de su fracción P-1 ó P-2 (80\%, $70 \%$ y $60 \%$, respectivamente).

Y realizando ahora los mismos cálculos numéricos con las respectivas probetas PUZC "hermanas" [70/30] y [60/40],

- $(0,4710 / 0,70)-[(0,7210-0,4072)+(0,6114-$ $0,3156)] / 2=0,3681 \mathrm{~g} \mathrm{SO}_{3} / \mathrm{l}$ (= contenido teórico) >> 0,0875 g SO $3 /$ (= content real), y

- $(0,4710 / 0,60)-[(0,7210-0,2225)+(0,6114-$ $0,1770)] / 2=0,3185 \mathrm{~g} \mathrm{SO}_{3} / \mathrm{l}$ (= contenido teórico) >> 0,0711 $\mathrm{g} \mathrm{SO}_{3} / \mathrm{l}$ (= contenido real).

Con lo que las correspondientes diferencias (el PUZC [P-1/MK 80/20]: 0,3677-0,1021 = 0,2656; el PUZC [P-1MK 70/30]: 0,3681 - 0,0875 =0,2806; y el PUZC [P-1/MK 60/40]: 0,3185-0,0711 = 0,2474), darían por resultado que según este otro parámetro químico

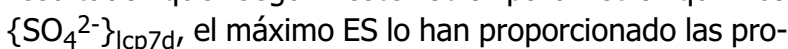
betas ASTM C 452-68 del PUZC [70/30] $(1,7)$, y no sus probetas "hermanas" [80/20] ni [60/40], como sí lo originó en cambio el POZC 60/40 con el método Le Chatelier-Ansttet (1, 4-6, 8).
Similarly, in the case of the SRPC PY-6: $\mathrm{SO}_{3} / \mathrm{I}=0.6114$ $\mathrm{g} \mathrm{SO}_{3} / \mathrm{l}-0.4008 \mathrm{~g} \mathrm{SO}_{3} / \mathrm{l}=0.2106 \mathrm{~g}$. Taking the mean of the two possible cases, it may be deduced that the $20 \%$ of $\mathrm{MK}$ has reacted with $0.2622 \mathrm{~g} \mathrm{SO}_{3} /$ l.

Employing now the same reasoning for plain $P-1$ $\left(14.11 \% C_{3} A\right)$, it may be deduced for its [80/20 P-1/MK] POZC variant that:

- If the MK behaves inertly (Inert SR material) there would be $0.4710 \times 100 / 80=0.58875 \mathrm{~g} \mathrm{SO}_{3} / \mathrm{l}(0.4710=$ $g \mathrm{SO}_{3} / \mathrm{l}$ in plain $\mathrm{P}-1$ at the age of 7 days) in the specimen storage water, and

- If the MK does not behave inertly but like a pozzolan, it would release $0.58875-0.22105=0.3677 \mathrm{~g} \mathrm{SO}_{3} / \mathrm{l}$ ( = theoretical value).

But neither of the above held; rather, the opposite was found to be true, namely that MK always behaves acts like a pozzolan. In fact, the $\left\{\mathrm{SO}_{4}{ }^{2-\}_{c w 7 d}}\right.$ content was three times smaller, i.e. $0.1021 \mathrm{~g} \mathrm{SO}_{3} / \mathrm{l}$ ( = real content), which is tantamount to saying that the $20 \%$ MK reacted three times more actively with the initial gypsum content $\left(15.05 \%=7.0 \% \mathrm{SO}_{3}\right)$ of its POZC than if it had been inert. In other words, it behaved not inertly but like a genuine pozzolan. Indeed, this substantial pozzolanic activity in the plaster-bearing solutions was what actually gave rise to the SE between the $\mathrm{Al}_{2} \mathrm{O}_{3}{ }^{r_{-}}$(present in the $20 \%, 30 \%$ or $40 \%$ $M K$ constituent) and the $C_{3} A$ (present in the $80 \%, 70 \%$ or $60 \%$ of the OPC constituent in $P-1$ or $P-2$ ) in each POZC.

Performing similar calculations for the respective POZC [70/30] and [60/40] specimens "siblings" yields the following:

- $(0.4710 / 0.70)-[(0.7210-0.4072)+(0.6114-$ $0.3156)] / 2=0.3681 \mathrm{~g} \mathrm{SO}_{3} / \mathrm{l}$ (= theoretical content) >> $0.0875 \mathrm{~g} \mathrm{SO}_{3} / \mathrm{l}$ ( = real content), and

- $(0.4710 / 0.60)-[(0.7210-0.2225)+(0.6114-$ $0.1770)] / 2=0.3185 \mathrm{~g} \mathrm{SO}_{3} / \mathrm{l}$ ( = theoretical content) >> $0.0711 \mathrm{~g} \mathrm{SO}_{3} / \mathrm{l}$ ( = real content).

The corresponding differences ((g/SO3/l) POZC [P-1/MK 80/20]: $0.3677-0.1021=0.2656 ;$ POZC [P-1/MK 70/30]: $0.3681-0.0875=0.2806$; and POZC [P-1/MK 60/40]: $0.3185-0.0711=0.2474$ ), have made that according to this chemical parameter, the highest SE has been originated by $[P-1 / M K$ 70/30] specimens. In other words, according to the ASTM C 452-68 test, the highest degree of SE was achieved for the [60/40 P-1/MK] specimens. By contrast, according to the values for $\left\{\mathrm{SO}_{4}{ }^{2-}\right\}_{\text {cw7d }}$ obtained for the ASTM C 452-68 specimens, under the initial assumption, the highest SE was observed in the [70/30 P1/MK] specimens but not in its "sibling" [80/20 P-1/MK] nor [60/40 P-1/MK] specimens. But when the POZC 60/40 was tested according to Le Chatelier-Ansttet gave rise to the maximum ESE $(1,4-6,8)$. 
En definitiva, el comportamiento real de las probetas, pero sobre todo de sus aguas de conservación, fue contrario al teórico, es decir, el contenido real de \{SO42\}lcp7d fue menor que su respectivo contenido teórico para todas las familias de PUZC $[\mathrm{P}-1 / \mathrm{MK}]$, [P-2/MK], [PY4/MK] y [PY-6/MK]. Además el contenido de \{SO42\}lcp7d disminuyó en toda la familia de PUZC cuando el contenido de MK aumentó. Por consiguiente, la suposición inicial no fue correcta, sino la contraria para todas las familias de PUZC.

$2^{\circ}$ supuesto: en este otro supuesto no se puede precisar la cantidad de $\mathrm{SO}_{3}$ del $7,0 \%$ inicial que la fracción de MK fijaría por sí sola en cada PUZC del CPRS PY-4 o del PY-6, De aquí que haya que centrarse en exclusiva en los correspondientes al cemento Pórtland matriz P-1 con el que también se había mezclado, en cuyo caso, véase la Tabla 4, se cumple una vez más a la edad de 7 días que, a más MK añadido, mayor cantidad se fija de su cantidad teórica P-1/Inerte ${ }_{\mathrm{RS}}$ correspondiente. Lo que indica también que se ha producido un ES, pero en este caso con el cemento de mezcla $60 / 40$ más que en el 70/30, como en el método L-A ( 1 , $4-6,8)$. Sin embargo, el mayor valor de ES no debe de deducirse del $1^{\circ}$ ni $2^{\circ}$ supuesto, porque la cuestión más importante es determinar el mayor o más apropiado ESE para cada objetivo, mediante los respectivos valores de $\Delta \mathrm{L}$ y $\mathrm{RM}$ que genera cada familia de PUZC. Continúese más adelante en el apartado 4.3. Consecuencias técnicas.

\subsection{Con los hormigones de cemento: probetas de hormigón picado y vibrado (con y $\sin 7,0 \%$ $\mathrm{SO}_{3}$ y conservadas en C.H. o bajo agua)}

\subsection{1. $\Delta \mathrm{Lxd}(\mathrm{mm} / \mathrm{m})$ originado por las probetas de hormigón después de haber sufrido ataque de los sulfatos $\left(=7,0 \% \mathrm{SO}_{3}\right)$}

El caso más favorable para demostrarlo se encuentra comparando las familias de PUZC P-1/MK y PY-6/MK junto con sus correspondientes CP puros P-1 y PY-6 (Figura 4). Así se tiene que:

4.2.1.1. El valor real de $\Delta \mathrm{L}_{7 \mathrm{~d}}(\mathrm{~mm} / \mathrm{m})$ determinado (de dos probetas $-10 \times 10 \times 50 \mathrm{~cm}-$ de hormigón vibrado por tipo de cemento)

$\left(\mathrm{a}_{1}\right)$ El valor de $\Delta \mathrm{L}_{7 \mathrm{~d}}$ de las probetas [PY-6/MK 60/40] es $=0,11 \mathrm{~mm} / \mathrm{m}$,

$\left(b_{1}\right)$ El valor de $\Delta \mathrm{L}_{7 \mathrm{~d}}$ de las probetas [PY-6] es 0,09; el $60 \%$ es $=0,054 \mathrm{~mm} / \mathrm{m}$,

$\left(c_{1}\right)$ El valor de $\Delta \mathrm{L}_{7 \mathrm{~d}}$ del $\left[40 \% \mathrm{MK}+7,0 \% \mathrm{SO}_{3}\right]=\left(\mathrm{a}_{1}\right)$ $-\left(b_{1}\right)=0,11-0,054=0,056 \mathrm{~mm} / \mathrm{m}$,

$\left(d_{1}\right)$ El valor de $\Delta L_{7 d}$ de las probetas $[P-1]$ es $0,38 \mathrm{~mm} / \mathrm{m}$; el $60 \%$ es $=0,228 \mathrm{~mm} / \mathrm{m}$.
In short, the real behavior of the specimens, and above all of their curing waters, was contrary to their theoretical behavior, i.e. the real $\left\{\mathrm{SO}_{4}{ }^{2-}\right\}_{\mathrm{cw} \text { dd }}$ content was always lower than the respective theoretical content for all the POZC in the $[P-1 / M K],[P-2 / M K],[P Y-$ $4 / M K]$ and $[P Y-6 / M K]$ families. Moreover, the $\left\{\mathrm{SO}_{4}{ }^{2-}\right.$ \}$_{c w 7 d}$ declined in all the POZC families when the MK content increased. Therefore, not the initial assumption but its opposite proved to be correct for all the POZC families.

Assumption 2: In this case, it is not possible to calculate the amount of the initial $7.0 \% \mathrm{SO}_{3}$ that the $\mathrm{MK}$ fraction would alone fix in each POZC from SRPC PY-4 or PY-6 origin. Consequently, the exercise must focus on the values for the POZC with OPC P-1, in which case the respective calculations yield the results given in Table 4. According to these results, the greater the amount of $M K$ added, more of the respective theoretical $P$-1/Inert $t_{S R}$ is fixed, which is also indicative of the existence of synergies; in this case, like in the L-A test, the synergies were more prominent in the [60/40] than in the [70/30] blended cement. However, the highest ESE value is not, under either the first or the second assumptions, the most important question, since it is the respective $\boldsymbol{\phi} L$ and MS values that indicate which POZC of each family generates the highest or most suitable ESE for each aim. See points 1 and 2 in 4.3. Technical Consequences, later on.

\subsection{Rodded and vibrated cement concrete specimens (with and without $7.0 \% \mathrm{SO}_{3}$ and stored in a moist closet or in water, respectively)}

\subsection{1. $\Delta L x d(\mathrm{~mm} / \mathrm{m})$ values after an aggressive sulfates attack $\left(=7.0 \% \mathrm{SO}_{3}\right)$}

The most suitable real method to prove the existence of ESE is by comparing 60/40 POZC P-1/MK and PY-6/MK and their plain PC $P-1$ and $P Y-6$, respectively (Figure 4).

\subsubsection{1. $\Delta L_{7 d}(\mathrm{~mm} / \mathrm{m})$ real value determined (by means of two specimens $-10 \times 10 \times 50 \mathrm{~cm}$ - of vibrated concrete by each cement type)}

(a $\left.{ }_{1}\right) \Delta L_{7 d}$ value for $[P Y-6 / M K]$ specimens is $=0.11 \mathrm{~mm} / \mathrm{m}$,

(b $\left.b_{1}\right) \Delta L_{7 d}$ value for $[P Y-6]$ specimens is $=0.09 \mathrm{~mm} / \mathrm{m}$; its $60 \%$ is $=0.054 \mathrm{~mm} / \mathrm{m}$,

(c $\left.c_{1}\right) \Delta L_{7 d}$ value for $\left[40 \% \mathrm{MK}+7.0 \% \mathrm{SO}_{3}\right]=\left(\mathrm{a}_{1}\right)-\left(b_{1}\right)$ $=0.11-0.054=0.056 \mathrm{~mm} / \mathrm{m}$,

(d $\left.d_{1}\right) \Delta L_{7 d}$ value for $[P-1]$ specimens is $=0.38 \mathrm{~mm} / \mathrm{m}$; its $60 \%$ is $=0.228 \mathrm{~mm} / \mathrm{m}$, 
$\left(e_{1}\right)$ El valor teórico de $\Delta \mathrm{L}_{7 \mathrm{~d}}$ de las probetas [P-1/MK $60 / 40]$ debe ser $=\left(d_{1}\right)+\left(c_{1}\right)=0,228+0,056=$ $0,284 \mathrm{~mm} / \mathrm{m}$, mientras que

$\left(f_{1}\right)$ El valor real de $\Delta L_{7 d}$ de las probetas [P-1/MK 60/40] ha sido $=0,75 \mathrm{~mm} / \mathrm{m}$, es decir, 2,64 veces mayor que el teórico $(=0,284 \mathrm{~mm} / \mathrm{m})$.

\subsubsection{El valor real de $\Delta \mathrm{L}_{10 \mathrm{~d}}(\mathrm{~mm} / \mathrm{m})$ determinado}

$\left(\mathrm{a}_{2}\right)$ El valor de $\Delta \mathrm{L}_{10 \mathrm{~d}}$ de las probetas [PY-6/MK 60/40] es $=0,39 \mathrm{~mm} / \mathrm{m}$,

$\left(b_{2}\right)$ el valor de $\Delta L_{10 d}$ de las probetas [PY-6] es 0,01 mm/m; el $60 \%$ es $=0,006 \mathrm{~mm} / \mathrm{m}$,

$\left(c_{2}\right)$ el valor de $\Delta \mathrm{L}_{10 d}$ del $\left[40 \% \mathrm{MK}+7,0 \% \mathrm{SO}_{3}\right]=\left(\mathrm{a}_{2}\right)$ $-\left(b_{2}\right)=0,39-0,006=0,384 \mathrm{~mm} / \mathrm{m}$,

$\left(\mathrm{d}_{2}\right)$ el valor de $\Delta \mathrm{L}_{10 \mathrm{~d}}$ de las probetas [P1] es 0,56 $\mathrm{mm} / \mathrm{m}$; el $60 \%$ es $=0,336 \mathrm{~mm} / \mathrm{m}$.

$\left(\mathrm{e}_{2}\right.$ ) el valor teórico de $\Delta \mathrm{L}_{10 \mathrm{~d}}$ de las probetas $[\mathrm{P}-1 / \mathrm{MK}$ $60 / 40]$ es $=\left(d_{2}\right)+\left(c_{2}\right)=0,336+0,384=0,720$ $\mathrm{mm} / \mathrm{m}$, mientras que,

$\left(f_{2}\right)$ el valor real de $\Delta L{ }_{10 d}$ de las probetas $[P-1 / M K$ $60 / 40]$ ha sido $=0,775 \mathrm{~mm} / \mathrm{m}$, es decir, 1,08 veces mayor que el teórico $(=0,720 \mathrm{~mm} / \mathrm{m})$.

En definitiva, los valores reales de $\Delta \mathrm{L}_{7 \mathrm{~d}}$ e $\Delta \mathrm{L}_{10 \mathrm{~d}}$ de las probetas [P-1/MK 60/40] resultaron ser 2,64 y 1,08 veces mayores que los teóricos como consecuencia del ESE de resultado adverso en este caso. Por tanto, ninguna de las dos familias de PUZC, P-1/MK ni PY-6/MK, se pueden considerar "cementos estables", desde el punto de vista de la resistencia al ataque de los sulfatos. Continúese más adelante en el apartado 4.3. Consecuencias técnicas, sólo que circunscritas únicamente en esta ocasión, a los CP puros P-1 y PY- 6 y a sus PUZC con MK.

\subsubsection{Resistencias mecánicas a compresión (RMC) y a tracción indirecta (RMTI)}

4.2.2.1. Analíticamente: RMC (48) y RMTI ("ensayo brasileño") (48) (Tablas 5 y 6 )

En general, a la edad de 7 días, los valores de $\mathrm{RMC}_{7 \mathrm{~d}}$ de las probetas de cada familia de PUZC [P-1/MK] y [PY$6 / M K]$ aumentaron con la cantidad de MK añadida. Así, el orden de tales valores de $\mathrm{RMC}_{7 \mathrm{~d}}$ guardó el siguiente orden: $\left(e_{1}\right)$ The theoretical $\Delta L_{7 d}$ value for $[P-1 / M K$ 60/40] specimens must be $=\left(d_{1}\right)+\left(c_{1}\right)=0.228+0.056$ $=0.284 \mathrm{~mm} / \mathrm{m}$, meanwhile,

$\left(f_{1}\right)$ The real $\Delta L_{7 d}$ value for [P-1/MK 60/40] specimens has been $=0.75 \mathrm{~mm} / \mathrm{m}$, i.e. 2.64-fold higher than its theoretical $\Delta L_{7 d}$ value $(=0.720 \mathrm{~mm} / \mathrm{m})$.

4.2.1.2. Real $\Delta L_{10 d}$ value $(\mathrm{mm} / \mathrm{m})$ determined:

(a $a_{2} \Delta L_{10 d}$ value for $[P Y-6 / M K]$ specimens is $=0.39$ $\mathrm{mm} / \mathrm{m}$,

(b) $\Delta L_{10 d}$ value for $[P Y-6]$ specimens is $=0.01 \mathrm{~mm} / \mathrm{m}$; its $60 \%$ is $=0.006 \mathrm{~mm} / \mathrm{m}$,

(c) $\Delta L_{10 d}$ value for $\left[40 \% \mathrm{MK}+7.0 \% \mathrm{SO}_{3}\right]=\left(\mathrm{a}_{2}\right)-\left(b_{2}\right)$ $=0.39-0.006=0.384 \mathrm{~mm} / \mathrm{m}$,

(d $\left.d_{2}\right) \Delta L_{10 d}$ value for $[P-1]$ specimens is $=0.56 \mathrm{~mm} / \mathrm{m}$; its $60 \%$ is $=0.336 \mathrm{~mm} / \mathrm{m}$,

$\left(e_{2}\right)$ The theoretical $\Delta L_{10 d}$ value for [P-1/MK 60/40] specimens must be $=\left(d_{2}\right)+\left(c_{2}\right)=0.336+0.384$ $=0.720 \mathrm{~mm} / \mathrm{m}$, meanwhile,

$\left(f_{2}\right)$ The real $\Delta L_{10 d}$ value for $[P-1 / M K 60 / 40]$ specimens has been $=0.775 \mathrm{~mm} / \mathrm{m}$, i.e. 1.08 -fold higher than its theoretical $\Delta L_{7 d}$ value $(=0.720 \mathrm{~mm} / \mathrm{m})$.

In short, at the ages of 7 and 10 days, then, ESE was also observed in the concrete specimens, inasmuch as the real $\Delta L_{7 d}(\mathrm{~mm} / \mathrm{m})$ value for $[60 / 40 \quad P-1 / \mathrm{MK}]$ specimens was from 2.64- to 1.08-fold higher than its theoretical $\boldsymbol{\Delta} \boldsymbol{L}_{\mathbf{7 d}}(\mathrm{mm} / \mathrm{m})$ value. Therefore, neither POZC family, $P-1 / M K$ or $P Y-6 / M K$, can be regarded as being "stable cements", in terms of resistance to sulfate attack. See point 2 in Section 4.3. Technical Consequences, later on.

\subsubsection{Compressive strength (CS) and tensile strength (ITS)}

4.2.2.1. Analytically: CS (48) and ITS (48) (Tables 5 an 6).

At the age of 7 days, the CS $_{7 d}$ values for the [P-1/MK] and $[P Y-6 / M K]$ specimens generally increased with the proportion of $M K$. The order of these $\mathrm{CS}_{7 d}$ values was as follows:

$$
<\mathrm{CS}_{7 \mathrm{~d}} \text { and } \mathrm{ITS}_{7 \mathrm{~d}}<[\mathrm{P}-1 / \mathrm{MK}] \text { and }[\mathrm{PY}-6 / \mathrm{MK}][100 / 00] \leq[80 / 20]<[70 / 30] \leq[60 / 40]
$$

Por tanto, la relación [1], (Ecuación [1]), es una consecuencia directa del efecto químico derivado de la sustitución física del CPO P-1 y del CPRS PY-6 por MK, es decir, la alúmina reactiva $\mathrm{Al}_{2} \mathrm{O}_{3}{ }^{\mathrm{r}-}$, presente en el $\mathrm{MK}$ cuando se convirtió en ett-rf (1-8). De aquí que para ambas familias de PUZC ensayadas, dicha ett-rf sea el principal
Hence, relationship [1], (Eq. [1]) is a consequence of the direct chemical effect deriving from the physical replacement of OPC $P-1$ and SRPC $P Y-6$ by $M K$, i.e., the $\mathrm{Al}_{2} \mathrm{O}_{3}{ }^{r-}$ present in $\mathrm{MK}$ when converted into ett-rf (1-8) prevailed. It follows that ett-rf is the chief agent involved in the processes leading to Eq. [1] for both POZC families 
agente implicado en el proceso que desemboca en la Ecuación [1]. Por el contrario, a la edad de 90 días, se obtuvo el orden o clasificación inversa, es decir: tested. At the age of 90 days, on the contrary, the order was reversed:

$$
\begin{gathered}
>\mathrm{CS}_{90 \mathrm{~d}} \text { and } \mathrm{ITS}_{90 \mathrm{~d}}>[\mathrm{P}-1 / \mathrm{MK}][80 / 20]>[70 / 30]>[60 / 40]>[100 / 00] \\
>\mathrm{CS}_{90 \mathrm{~d}}>[\mathrm{PY}-6 / \mathrm{MK}][80 / 20]>[70 / 30]>[60 / 40]>[100 / 00]
\end{gathered}
$$

En otras palabras, en este otro caso, los que prevalecieron principalmente fueron los contenidos de $\mathrm{C}_{3} \mathrm{~S}$ y $\mathrm{C}_{2} \mathrm{~S}$, de los CP P-1 y PY-6 (Ecuaciones [2] y [3]) (Tabla 6).

Una prueba adicional encontrada que corrobora la veracidad de la hipótesis de comportamiento aducida, pero en particular la segunda, fue la siguiente:

- Por una parte y de acuerdo con los valores de $\mathrm{TI}_{90 \mathrm{~d}}$ de la familia de probetas [PY-6/MK], de nuevo y una vez más, se debe de obtener el orden o clasificación inicial o Ecuación [1], y

- Por otra parte, en ausencia del $7,0 \% \mathrm{SO}_{3}$, se debe de obtener también el orden o clasificación contraria, es decir, las Ecuaciones [2] o [3]; además, si la fracción del $C P$ no posee nada de $C_{3} A$, caso de las probetas de la familia de PUZC PY-6/MK, se debe de preservar el orden o clasificación inicial o Ecuación [1] con ambos parámetros de resistencia mecánica; pero si el $\mathrm{CP}$ posee $\mathrm{C}_{3} \mathrm{~A}$, caso de los $\mathrm{PUZC}$ de la familia $\mathrm{P}-1 / \mathrm{MK}$, el orden inicial deberá preservarse mejor con el parámetro $\mathrm{RMTI}_{28 \mathrm{~d}}$ que con el parámetro $\mathrm{RMC}_{28 \mathrm{~d}}$.

Y tales clasificaciones o Ecuaciones [4], [5], [6] y [7], respectivamente, se han obtenido, es decir:
In other words, the $C_{3} S$ and $C_{2} S$ in P-1 and PY-6 PC, respectively, prevailed (Eqs. [2] and [3]) (Table 6). Further evidence was found that corroborated both behavioral hypotheses, but in particular the second, namely:

- on the one hand and according to ITS ${ }_{90 d}$ for the [PY$6 / M K]$ family specimens, once again, the initial order, Eq. [1], must take place, and

- on the other hand, in the absence of $7.0 \% \mathrm{SO}_{3}$, the reverse order, Eqs.[2] or [3], must take place as well; furthermore, if the $P C$ fraction has no $C_{3} A, P Y-6 / M K$ family specimens, the initial order, Eq. [1], must not be preserved with both mechanical parameters, but if it has $C_{3} A, P-1 / M K$ family specimens, it must be preserved with $I T S_{28 d}$ parameter better than with $\mathrm{CS}_{28 d}$ parameter.

And such classifications or Eqs. [4], [5], [6] and [7], respectively, have been obtained, i.e.

$$
\begin{gathered}
<\mathrm{RMTI}_{90 \mathrm{~d}}<[\mathrm{PY}-6 / \mathrm{MK}][100 / 00]<[80 / 20]<[70 / 30]<[60 / 40] \\
>\mathrm{RMC}_{28 \mathrm{~d}} \text { Y RMTI }_{28 \mathrm{~d}}>\mathrm{PY}-6 / \mathrm{MK} 80 / 20>70 / 30>60 / 40 \\
<\mathrm{RMC}_{28 \mathrm{~d}}<\mathrm{P}-1 / \mathrm{MK} 80 / 20<70 / 30<60 / 40 \\
>\mathrm{RMTI}_{28 \mathrm{~d}}>\mathrm{P}-1 / \mathrm{MK} 80 / 20>70 / 30>60 / 40
\end{gathered}
$$

\subsubsection{Numéricamente: $\mathrm{RMC}_{28 \mathrm{~d}}$}

(a) La $\mathrm{RMC}_{28}$ de las probetas [PY-6/MK 80/20] es $251,0 \mathrm{Kp} / \mathrm{cm}^{2}$; el $80 \%$ es atribuido al CPRS PY-6 = $200,8 \mathrm{Kp} / \mathrm{cm}^{2}=\left(\mathrm{a}_{1}\right)$, y el $20 \%$ al MK con $7,0 \% \mathrm{SO}_{3}$ $=50,2 \mathrm{Kp} / \mathrm{cm}^{2}=\left(a_{2}\right)$,

(b) La $\mathrm{RMC}_{28}$ de las probetas [PY-6/MK 70/30] es $274,0 \mathrm{Kp} / \mathrm{cm}^{2}$; el $70 \%$ es atribuido al CPRS PY-6 = $191,8 \mathrm{Kp} / \mathrm{cm}^{2}=\left(\mathrm{b}_{1}\right)$, y el $30 \%$ al $\mathrm{MK}$ con $7,0 \% \mathrm{SO}_{3}$ $=82,2 \mathrm{Kp} / \mathrm{cm}^{2}=\left(\mathrm{b}_{2}\right)$,

(c) La $\mathrm{RMC}_{28}$ de las probetas [PY-6/MK 60/40] es $310,0 \mathrm{Kp} / \mathrm{cm}^{2}$; el $60 \%$ es atribuido al CPRS = $186,0 \mathrm{Kp} / \mathrm{cm}^{2}=\left(\mathrm{c}_{1}\right)$, y el $40 \%$ al $\mathrm{MK}$ con $7,0 \%$ $\mathrm{SO}_{3}=124,0 \mathrm{Kp} / \mathrm{cm}^{2}=\left(\mathrm{c}_{2}\right), \mathrm{y}$

\subsubsection{Numerically: $\boldsymbol{C S}_{\mathbf{2 8 d}}$.}

(a) $\mathbf{R M C}_{28}$ value for [PY-6/MK 80/20] specimens is 251.0 $\mathrm{Kp} / \mathrm{cm}^{2}$; its $80 \%$ would be from the SRPC PY- 6 origin $=200.8 \mathrm{Kp} / \mathrm{cm}^{2}=\left(a_{1}\right)$, and its $20 \%$ would be from the $20 \% \mathrm{MK}$ with $7.0 \% \mathrm{SO}_{3}$ origin $=50.2 \mathrm{Kp} / \mathrm{cm}^{2}=\left(\mathrm{a}_{2}\right)$,

(b) $\boldsymbol{R M C}_{28}$ value for [PY-6/MK 70/30] specimens is 274.0 $\mathrm{Kp} / \mathrm{cm}^{2}$; its $70 \%$ would be from the SRPC PY-6 origin $=191.8 \mathrm{Kp} / \mathrm{cm}^{2}=\left(\mathbf{b}_{1}\right)$, and its $30 \%$ would be from the $30 \% \mathrm{MK}$ with $7.0 \% \mathrm{SO}_{3}$ origin $=82.2 \mathrm{Kp} / \mathrm{cm}^{2}=\left(b_{2}\right)$,

(c) $\mathbf{R M C}_{28}$ value for [PY-6/MK 60/40] specimens is 310.0 $\mathrm{Kp} / \mathrm{cm}^{2}$; its $60 \%$ would be from the SRPC PY-6 origin $=186.0 \mathrm{Kp} / \mathrm{cm}^{2}=\left(c_{1}\right)$, and its $40 \%$ would be from the $40 \% \mathrm{MK}$ with $7.0 \% \mathrm{SO}_{3}=124.0 \mathrm{Kp} / \mathrm{cm}^{2}=\left(c_{2}\right)$, and 
(d) La RMC 28 de las probetas [P-1] es $221,0 \mathrm{Kp} / \mathrm{cm}^{2}$; el $80 \%$ es $=176,8 \mathrm{Kp} / \mathrm{cm}^{2}=\left(\mathrm{d}_{1}\right)$, el $70 \%=154,7$ $\mathrm{Kp} / \mathrm{cm}^{2}=\left(\mathrm{d}_{2}\right)$, y el $60 \%=132,6 \mathrm{Kp} / \mathrm{cm}^{2}=\left(\mathrm{d}_{3}\right)$.

Sumando ordenadamente los correspondientes valores anteriores, se obtienen los siguientes resultados:

- El valor teórico de $\mathrm{RMC}_{7 \mathrm{~d}}$ de las probetas $[\mathrm{P}-1 / \mathrm{MK}$ $80 / 20]$ sería $\left(\left(d_{1}\right)+\left(a_{2}\right)\right)=176,8+50,2=227,0$ $\mathrm{Kp} / \mathrm{cm}^{2}$, mientras que su valor real ha sido 231,0 $\mathrm{Kp} / \mathrm{cm}^{2}$, el cual es $>227,0 \mathrm{Kp} / \mathrm{cm}^{2}$, siendo la diferencia de $4,0 \mathrm{Kp} / \mathrm{cm}^{2}$,

- El valor teórico de $\mathrm{RMC}_{7 \mathrm{~d}}$ de las probetas[P-1/MK $70 / 30]$ sería $\left(\left(d_{2}\right)+\left(b_{2}\right)\right)=154,7+82,2=236,9$ $\mathrm{Kp} / \mathrm{cm}^{2}$, mientras su valor real ha sido $266,0 \mathrm{Kp} / \mathrm{cm}^{2}$, el cual es $>236,9 \mathrm{Kp} / \mathrm{cm}^{2}$, siendo la diferencia de $29,7 \mathrm{Kp} / \mathrm{cm}^{2}, y$

- El valor teórico de $\mathrm{RMC}_{7 \mathrm{~d}}$ de las probetas $[\mathrm{P}-1 / \mathrm{MK}$ $60 / 40]$ sería $\left(\left(d_{3}\right)+\left(c_{2}\right)\right)=132,6+124,0=256,6$ $\mathrm{Kp} / \mathrm{cm}^{2}$, mientras su valor real $\mathrm{RMC}_{7 \mathrm{~d}}$ ha sido 266,0 $\mathrm{Kp} / \mathrm{cm}^{2}$, el cual es $>256,6 \mathrm{Kp} / \mathrm{cm}^{2}$, siendo la diferencia de $9,4 \mathrm{Kp} / \mathrm{cm}^{2}$,

En definitiva, a la edad de 7 días, el valor real de $\mathrm{RMC}_{7 \mathrm{~d}}$ de las probetas de hormigón de los PUZC de la familia [P-1/MK] ha sido $1,02,1,12$ y 1,04 veces mayor que sus correspondientes valores teóricos, como consecuencia del ESE originado de resultado no adverso en este caso, si bien y a pesar de que todos sus valores de $\mathrm{RMC}_{7 \mathrm{~d}}$ sean semejantes a los de cualquier CPRS, su falta de la necesaria estabilidad de volumen frente al ataque del yeso por causa del MK y del propio CPO P-1, los menoscaban seriamente hasta el punto de no poderlos considerar a ninguno de ellos tan estable como un CPRS ante dicho ataque agresivo. Continúese más adelante en el apartado 4.3. Consecuencias técnicas, sólo que circunscritas únicamente a los CP puros P-1 y PY-6 y a sus PUZC P-1/MK y PY-6/MK. Además,

(e) El valor de $\mathrm{RMC}_{7 \mathrm{~d}}$ de las probetas del CPRS [PY-6 $100 / 00]$ es $226,0 \mathrm{Kp} / \mathrm{cm}^{2}$; el $80 \%$ es $=180,8$ $\mathrm{Kp} / \mathrm{cm}^{2}$, el $70 \%$ es $=158,2 \mathrm{Kp} / \mathrm{cm}^{2}$, y el $60 \%$ es $=$ $135,6 \mathrm{Kp} / \mathrm{cm}^{2}$,

(f) El $80 \% x R M C_{7 d}\left(=251,0 \mathrm{Kp} / \mathrm{cm}^{2}\right)$ de las probetas [PY-6/MK 80/20] es $=200,8 \mathrm{Kp} / \mathrm{cm}^{2}$, es decir > $180,8 \mathrm{Kp} / \mathrm{cm}^{2}$, siendo la diferencia de $20,0 \mathrm{Kp} / \mathrm{cm}^{2}$,

(g) El $70 \% \times \mathrm{XMCC}_{7 \mathrm{~d}}\left(=274,0 \mathrm{Kp} / \mathrm{cm}^{2}\right)$ de las probetas [PY$6 / \mathrm{MK} 70 / 30]$ es $=191,8 \mathrm{Kp} / \mathrm{cm}^{2}$, es decir $>158,2$ $\mathrm{Kp} / \mathrm{cm}^{2}$, siendo la diferencia de $33,6 \mathrm{Kp} / \mathrm{cm}^{2}$, y

(h) El $60 \% \times x C_{7 d}\left(=310,0 \mathrm{Kp} / \mathrm{cm}^{2}\right)$ de las probetas [PY-6/MK 60/40] es $=186,0 \mathrm{Kp} / \mathrm{cm}^{2}$, es decir > $135,6 \mathrm{Kp} / \mathrm{cm}^{2}$, siendo al diferencia de $50,4 \mathrm{Kp} / \mathrm{cm}^{2}$.

En breve, el valor absoluto de las diferencias aumentó con la cantidad de MK añadida; es decir, la causa de dicho aumento ha de serle imputada al MK con 7,0\% (d) $R_{M} C_{28}$ value for [P-1] specimens is $221.0 \mathrm{Kp} / \mathrm{cm}^{2}$; its $80 \%$ is $=176.8 \mathrm{Kp} / \mathrm{cm}^{2}=\left(d_{1}\right)$, its $70 \%=154.7$ $\mathrm{Kp} / \mathrm{cm}^{2}=\left(\boldsymbol{d}_{2}\right)$, and its $60 \%=132.6 \mathrm{Kp} / \mathrm{cm}^{2}=\left(\boldsymbol{d}_{3}\right)$.

Finally, summing orderly, the following results are obtained, namely:

- Theoretical $R M C_{7 d}$ value for [P-1/MK 80/20] specimens would be $\left(\left(\mathbf{d}_{1}\right)+\left(\mathbf{a}_{2}\right)\right)=176.8+50.2=$ $227.0 \mathrm{Kp} / \mathrm{cm}^{2}$, while its real $\mathbf{R M C}_{\mathbf{7 d}}$ value has been $231.0 \mathrm{Kp} / \mathrm{cm}^{2}$, which is $>227.0 \mathrm{Kp} / \mathrm{cm}^{2}$, being the difference $4.0 \mathrm{Kp} / \mathrm{cm}^{2}$,

- Theoretical $R M C_{7 d}$ value for the $[P-1 / M K$ 70/30] specimens would be $\left(\left(\boldsymbol{d}_{\mathbf{2}}\right)+\left(\boldsymbol{b}_{\mathbf{2}}\right)\right)=154.7+82.2=$ $236.9 \mathrm{Kp} / \mathrm{cm}^{2}$, while its real $\mathbf{R M C}_{\mathbf{7 d}}$ value has been $266.0 \mathrm{Kp} / \mathrm{cm}^{2}$, which is $>236.9 \mathrm{Kp} / \mathrm{cm}^{2}$, being the difference $29.7 \mathrm{Kp} / \mathrm{cm}^{2}$, and

- Theoretical $R M C_{7 d}$ value for [P-1/MK 60/40] specimens would be $\left(\left(\boldsymbol{d}_{3}\right)+\left(\boldsymbol{c}_{2}\right)\right)=132.6+124.0=$ $256.6 \mathrm{Kp} / \mathrm{cm}^{2}$, while its real $\mathbf{R M C}_{\mathbf{7 d}}$ value has been $266.0 \mathrm{Kp} / \mathrm{cm}^{2}$, which is $>256.6 \mathrm{Kp} / \mathrm{cm}^{2}$, being the difference $9.4 \mathrm{Kp} / \mathrm{cm}^{2}$.

In short, by the age of 7 days, then, ESE has again taken place with beneficial consequences for the cement concrete, inasmuch as the real $\mathbf{C S}_{\mathbf{2 8 d}}$ values for the concrete manufactured with [P-1/MK] family POZC were 1.02-, 1.12- and 1.04-fold higher than the respective theoretical $\mathbf{C S}_{\mathbf{2 8 d}}$ values. Nonetheless, [P-1/MK] family POZC can not be regarded as being "stable cements", in terms of resistance to sulfate attack, although their CS values are similar to the pattern observed in any SRPC. See point 2 in Technical Consequences, later on. Moreover,

(e) $\mathrm{CS}_{7 d}$ for plain [100/00 PY-6] specimens is 226.0 $\mathrm{Kp} / \mathrm{cm}^{2}$; the $80 \%$ value is $=180.8 \mathrm{Kp} / \mathrm{cm}^{2}$, the $70 \%$ value is $=158.2 \mathrm{Kp} / \mathrm{cm}^{2}$, and the $60 \%$ value is = $135.6 \mathrm{Kp} / \mathrm{cm}^{2}$,

(f) $80 \% x C S_{7 d}\left(=251.0 \mathrm{Kp} / \mathrm{cm}^{2}\right)$ for the $[80 / 20 \mathrm{PY}-6 / \mathrm{MK}]$ specimens $=200.8 \mathrm{Kp} / \mathrm{cm}^{2}$, i.e. $>180.8 \mathrm{Kp} / \mathrm{cm}^{2}$, the difference being $20.0 \mathrm{Kp} / \mathrm{cm}^{2}$,

(g) $70 \% x \mathrm{CS}_{7 d}\left(=274.0 \mathrm{Kp} / \mathrm{cm}^{2}\right)$ for the $[70 / 30 \mathrm{PY}$ $6 / \mathrm{MK}]$ specimens $=191.8 \mathrm{Kp} / \mathrm{cm}^{2}$, i.e. $>158.2$ $\mathrm{Kp} / \mathrm{cm}^{2}$, the difference being $33.6 \mathrm{Kp} / \mathrm{cm}^{2}$, and

(h) $60 \% x \mathrm{CS}_{7 d}\left(=310.0 \mathrm{Kp} / \mathrm{cm}^{2}\right)$ for the $[60 / 40 \mathrm{PY}$ $6 / \mathrm{MK}]$ specimens $=186.0 \mathrm{Kp} / \mathrm{cm}^{2}$, i.e. $>135.6$ $\mathrm{Kp} / \mathrm{cm}^{2}$, the difference being $50.4 \mathrm{Kp} / \mathrm{cm}^{2}$.

In short, absolute values of the differences increased with $M K$; i.e. the cause of the rise was the $M K$ with $7.0 \%$ $\mathrm{SO}_{3}$, or more precisely, the $\mathrm{Al}_{2} \mathrm{O}_{3}{ }^{r-}$ present in $\mathrm{MK}$ and 
$\mathrm{SO}_{3}$, o más exactamente, la cantidad de $\mathrm{Al}_{2} \mathrm{O}_{3}{ }^{\mathrm{r}}$ - del $\mathrm{MK}$ fue la responsable, y por este motivo, la formación de ett-rf prevaleció a edades iniciales (hasta los 7 días) (1-11); de acuerdo con este comportamiento, la consecuencia práctica de la rápida formación de ettringita es beneficiosa. Como pruebas adicionales que corroboran la última afirmación están las siguientes:

- lógicamente, las relaciones encontradas no fueron como en la Ecuación [2], sino la opuesta o Ecuación [1], pero sobre todo

- cuando esta misma familia de PUZC P-1/MK se ensayó sin el 7,0\% $\mathrm{SO}_{3}$, no mostró evidencia alguna de producir ESE, pero cuando se ensayaron con $45,16 \%$ yeso $\left(=21,0 \% \mathrm{SO}_{3}\right.$ en lugar de $7,0 \%$ ), sus probetas de hormigón picado 70/30 y 60/40, se auto-destruyeron por ataque del yeso un año y medio antes que las de su CPO puro P-1 $(1,6)$, es decir, una consecuencia adversa, técnicamente hablando; este efecto tan nocivo derivado del ataque del yeso se realizó en tal medida y con tanta rapidez, que pudo ser denominado con fundamento "ataque rápido del yeso" (1, $4,5,10,11,14)$, y con probetas de hormigón de 1 a 1,5 años antes (14).

\subsection{Consecuencias técnicas}

1. En otras palabras, a diferencia de los CPRS, los PUZC P-1/MK, P-2/MK, PY-4/MK, PY-6/MK 80/20, 70/30 y $60 / 40$, no se pueden considerar "cementos estables", desde el punto de vista de su resistencia a los sulfatos.

2. No obstante y por causa del MK precisamente que contienen, con $7,0 \% \mathrm{SO}_{3}$ y un apropiado curado hídrico previo, algunos de ellos pueden ser considerados "cementos hidráulicos expansivos" (51). Otra consecuencia beneficiosa sería también, que con otro contenido adecuado de $\mathrm{SO}_{3}$ pero menor que el $7,0 \%$, dicho PUZC podrá ser catalogado como "cemento sin retracción" (esta otra cantidad adecuada de $\mathrm{SO}_{3}$ también en exceso, le proporcionaría a dicho PUZC una expansión tal que compensara su retracción hidráulica, térmica, por secado, etc.), y si la cantidad es aún menor todavía, podrá ser catalogado como PUZC. Las tres cantidades adecuadas pero diferentes de $\mathrm{SO}_{3}$, para que dicho PUZC pueda ser catalogado de "cemento hidráulico expansivo", "cemento sin retracción" osimplemente PUZC, se determinan también mediante el método ASTM C 452-68 adaptado por R. Talero $(1,52)$, cuyos fundamentos químico-físicos son muy diferentes al del "óptimo de regulador de fraguado" de un CP puro (53). Dichas tres cantidades "óptimas" de regulador de fraguado de cada uno de ellos habrán de disminuir su valor absoluto en dicho orden, hasta el punto que la del último ó PUZC, incluso habrá de ser obligatoriamente $\leq 3,5 \%$ o $\leq 4,0 \%$, según que dicho PUZC sea cemento Tipo II o Tipo IV o V, respectivamente (54). responsible for the rapid formation of ett-rf prevailed at early ages (up to 7 days) (1-11); according to these findings, then, the practical implication of the rapid formation of ettringite are beneficial. Further findings that corroborate the latter assertion are:

- logically, the relationships found were not as in Eq. [2], but just the opposite, as in Eq. [1], but above all,

- when tested without $7.0 \% \mathrm{SO}_{3}$, this same $\mathrm{P}-1 / \mathrm{MK}$ family POZC showed no evidence of ESE, and when tested with $45.16 \%\left(=21.0 \% \mathrm{SO}_{3}\right.$ for the $\mathrm{RT}-86: \Delta \mathrm{L}$ test), they were destroyed by gypsum attack 3 months earlier than their plain OPC P-1 (Table 5), an adverse consequence, technologically speaking; this detrimental effect derived from gypsum attack was to such an extent and fast that it could be described as "rapid gypsum attack" (1, 4, 5, 10, 11, 14). And with concrete specimens, from 1 to 1.5 years earlier (14).

\subsection{Technical Consequences}

1. In other words, unlike SRPC, 80/20, 70/30 and 60/40 $P-1 / M K, P-2 / M K, P Y-4 / M K$ and PY-6/MK POZC can not be regarded as being "stable cements" in terms of resistance to sulfate attack.

2. Nonetheless and just due to the $\mathrm{MK}$, with $7.0 \% \mathrm{SO}_{3}$ ( = $15.05 \%$ gypsum) and suitable prior curing water, some can be regarded as being "expansive hydraulic cements" (51), and if the adequate content of $\mathrm{SO}_{3}$ is lesser than $7.0 \%$, some of them can be regarded as being "cement without shrinking" (this another appropriated $\mathrm{SO}_{3}$ content, in excess too, would give a this new POZC an expansion similar to its hydraulic, thermal, by drying, etc. shrinking), and if such $\mathrm{SO}_{3}$ content is still smaller, this cement will be able to be regarded as being POZC only (according to the $\Delta L v s$. Time originated by their respective specimens and determined and quantified by the ASTM C 452-68 method adapted by $R$. Talero $(1,52)$, whose fundamentals are very different to the "setting regulator optimum" of any plain clinker Portland (53). These three "optimum amounts" of setting regulator must be decreased in its absolute value according to the mentioned order, including and up to the point, that the last one POZC of these three cases has to be mandatory lower and undergoes the following conditions, $\leq 3.5 \%$ or $\leq 4.0 \%$, fulfilled in function of the cement POZC type considered: Type II or Type IV or Type V (54), respectively. 
3. En cambio, si la cantidad de yeso añadida en exceso a estos PUZC es mayor que el 7,0\% $\mathrm{SO}_{3}$, la consecuencia deja de ser beneficiosa para tornarse en perjudicial -"ataque rápido del yeso"-, y tanto más perjudicial, cuanto más mayor es aún que $7,0 \% \mathrm{SO}_{3}$ $(1,4,5,10,14)$. Así y cuando fue $21,0 \%$, las probetas de la familia de PUZC $\mathrm{P}-1 / \mathrm{MK}$ se destruyeron por ataque sulfático, 3 meses antes que las probetas de su propio CP P-1 puro (Tabla 7). Y con probetas de hormigón picado, de 1 a 1,5 años más pronto (14).
3. In contrast, if the amount of gypsum added in excess to these POZC is greater than $7.0 \%$, the consequence is not beneficial but very harmful, and the more harmful is, the more amount of gypsum in excess is added. To such a point that, the sulphate attack is so much intense, aggressive and fast, and for this reason, it justified to be named "rapid gypsum attack" $(1,4,5,10,11,14)$. Thus and when its $\mathrm{SO}_{3}$ content was $21.0 \%$, the POZC family $P-1 / \mathrm{MK}$ specimens were destroyed by sulphate attack, 95 days before than the specimens of its plain OPC $P$ 1 (Table 7). And with specimens of rodded concrete, 1.5 years earlier (14).

Tabla 7 / Table 7

Ensayo RT-86: $\Delta \mathrm{L}\left(\approx\right.$ al ensayo ASTM C $452-68$ pero con $21,0 \% \mathrm{SO}_{3}$ en lugar de $\left.7,0 \%\right): \Delta \mathrm{L}(\%)$. Resultados de algunos cementos. $R T-86: \Delta L$ test ( $\approx$ ASTM C $452-68$ test but with $21.0 \% \mathrm{SO}_{3}$ instead of $\left.7.0 \%\right): \Delta L(\%)$. Results of some cement.

\begin{tabular}{|c|c|c|c|c|}
\hline \multicolumn{5}{|c|}{$\Delta \mathrm{L}^{*}$ xdays $(\%)$ de dos CP y de sus PUZC con MK / $\Delta L^{*}$ xdays $(\%)$ for two $P C$ and for their POZC with $M K$} \\
\hline \multirow{2}{*}{ Edad (días) / Age (days) } & \multirow{2}{*}{$\begin{array}{c}\mathrm{CPO} / \mathrm{OPC} \\
\mathrm{P}-1\end{array}$} & \multirow{2}{*}{$\begin{array}{c}\text { CPRS / SRPC } \\
\text { PY-6 } \\
\cdots\end{array}$} & \multicolumn{2}{|c|}{ PUZC con MK / POZC with MK } \\
\hline & & & P-1/MK 60/40 & PY-6/MK 80/20 \\
\hline 7 & 0.043 & 0.005 & 0.575 & 0.036 \\
\hline 14 & 0.079 & 0.006 & 0.981 & 0.065 \\
\hline 21 & 0.120 & 0.008 & 1.119 & 0.082 \\
\hline 28 & 0.157 & 0.009 & 1.279 & 0.106 \\
\hline 60 & 1.229 & 0.013 & 1.366 & 0.149 \\
\hline 90 & 2* & 0.014 & 1.618 & 0.209 \\
\hline 120 & & 0.016 & 1.716 & 0.276 \\
\hline 150 & & 0.018 & 1.822 & 0.372 \\
\hline 180 & & 0.019 & 2* & 0.494 \\
\hline 270 & & 0.019 & 3* & $2 *$ \\
\hline 365 & 3* & 0.019 & & 3* \\
\hline 545 & & 0.021 & & \\
\hline 730 & 3* & 0.023 & 3* & 3* \\
\hline
\end{tabular}

* Los valores de $\triangle \mathrm{L}(\%)$ del resto de CPO, CPRS y sus PUZC con MK han sido publicados hace años $(1,12,14)$. / $\triangle L(\%)$ values for the rest of OPC, SRPC and their POZC, were published years ago $(1,12,14)$.

$2^{*}$ Probetas inmedibles porque estaban muy curvadas / Measureless specimens because they were much curved.

$3^{*}$ El mortero original con el que se fabricaron las probetas, estaba totalmente deshecho y dispersado en su agua de conservación. / Original mortar that the specimens had been manufactured with, was totally unmade and later on dispersed into its curing water.

\subsection{Mediante el ensayo de Emisión Acústica}

Este ensayo no destructivo (END) está basado en el ultrasonido (49) pero usando el parámetro energía (50) en lugar del parámetro velocidad propiamente dicho (49). Las EA son vibraciones mecánicas transitorias generadas por una rápida vibración de energía cuyo origen se localizado dentro de ciertos materiales (cristales piezo-eléctricos, por ejemplo). El valor medio normalizado del espectro de energía producido se computa del siguiente modo (ecuación [8]):

$$
E=\int_{w i}^{w p}|A(w)|^{2} d w / 2 \pi\left(w_{p}-w_{i}\right) A\left(w_{p}\right)^{2}
$$

Donde $\mathrm{E}=$ el valor medio del espectro de energía normalizado; $\mathrm{A}(\mathrm{w})$ = la amplitud de la frecuencia y $\mathrm{w}_{i}$ y $\mathrm{w}_{p}$

\subsection{By the Acoustic Emission test}

This non-destructive test is based in the Pulse Velocity test (49) but using energy parameter (50) instead of pulse velocity parameter properly (49). Acoustic emissions are transient mechanical vibrations generated by rapid release of energy from localized sources within materials (piezo-electric crystals, for instance). The normalized average spectral energy of $\boldsymbol{A E}$ events is computed from the following relationship (Eq. [8]):
Where $\boldsymbol{E}=$ normalized average spectral energy; $\boldsymbol{A}(\boldsymbol{w})=$ frequency amplitude and $\boldsymbol{w}_{\boldsymbol{i}}$ and $\boldsymbol{w}_{\boldsymbol{p}}$ correspond to the 
corresponden a las frecuencias inicial y máxima o del pico mayor. La Figura 5 representa el conjunto acumulado del espectro de energía en función del número de emisiones acústicas producidas. La energía del espectro muestra una relación bi-lineal separando los primeros estadios a fases del hormigón de su estado o expansión final que se le origina en el caso de las probetas de los CP P-1 y PY-6 y de sus PUZC con MK. Por otra parte, el conjunto de energía espectral proporciona información detallada acerca del incremento de longitud sufrido por las probetas, de modo y manera que el espectro del haz de ondas de EA recogido en el receptor es propio de cada hormigón de PUZC, así como también, de la geometría y estado de "salud" o "daño" sufrido por sus probetas. De aquí que esta técnica sea más eficiente como END que cualquier otra para asegurar la cuantía del daño que este tipo de puzolanas alumínicas en carácter químico, como el MK de este trabajo y el de otros previos (1-11, 14, 17-33), pueden llegar a originarle al hormigón del que formen parte, frente al ataque del yeso.

Por último, la razón por la que sea cual fuere el parámetro afín que se considere, el factor multiplicador o potenciador que ha justificado el ESE ha sido siempre más pequeño en el caso de los hormigones que en el caso de los correspondientes morteros, ha de buscarse necesariamente en que, a igualdad de todo lo demás, el sistema de macroporos, poros, microporos y capilares del hormigón es siempre mayor que el del correspondiente mortero (y mayor aún que el de su pasta, lógicamente). Lo que promueve a que la innata expansividad de toda la ettringita de cualquier origen que se forme en su interior durante su hidratación selenitosa, se invierta bastante más en rellenar dicho sistema de macroporos, poros, microporos y capilares del hormigón que en hacer que expanda. Puesto que en este último caso, la probabilidad de que toda la ettringita que se forme de cualquiera de estos dos orígenes: $\mathrm{Al}_{2} \mathrm{O}_{3}{ }^{\mathrm{r}-}$ del $\mathrm{MK}$ y $\mathrm{C}_{3} \mathrm{~A}$ del $\mathrm{CP}$, lo haga siempre enfrente de una partícula de árido es menor.

\section{CONCLUSIONES}

1. Ha quedado demostrado que sea cual fuere el ensayo y parámetro considerado, la formación conjunta en un medio selenitoso común (co-precipitación), hormigón o mortero de cemento, de la ettringita de origen $\mathrm{Al}_{2} \mathrm{O}_{3}{ }^{\mathrm{r}-}$ de las puzolanas, y la de origen $\mathrm{C}_{3} \mathrm{~A}$ de los $\mathrm{CPO}$, ha resultado ser siempre, cuantitativamente hablando, más que aditiva, sinérgica.

2. Sea cual fuere el parámetro o ensayo que se considere, y desde el punto de vista técnico, las consecuencias del Efecto Sinérgico Expansivo entre ambas ettringitas pueden ser calificadas como "beneficiosas", "adversas"o "indiferentes". A tal efecto, cabe la posibilidad de que tales calificativos no se puedan mantener si se consideran initial and peak frequencies. Figure 5 represents the accumulative plot of the spectral energy as a function of the $\boldsymbol{A E}$ events. The spectral energy exhibits a bilinear relationship separating the first expansions and the ultimate states of expansion for OPC $P-1$ and for SRPC $P Y-6$ and for their POZC with MK. A cumulative plot of the spectral energy provides deterministic information about the increase in length of the specimens. The spectral energy of an $\boldsymbol{A E}$ event is a function of the spectral signature of the individual POZC concrete as well as the geometry and the state of "health" or damage of the surrounding "composite". As a result, this technique is most efficient for NDE and damage assessment in POZC concretes with "aluminic" pozzolans in chemical character (1-11, 14, 17-33), such as this MK, which are placed in front of gypsum attack.

Finally and regardless of the related evaluative parameter considered, the multiplier-factor that justifies the ESE is always smaller for any concrete than for its respective mortar, since the macroporous, porous, microporous and capillary system in concrete is always greater, and still greater than for its cement paste, obviously. In consequence, the inborn ettringita expansion, formed from any origin during the sulphatic hydration, gives rise to an increase in length of the specimen and, in parallel, to fill full its mentioned macroporous, porous, microporous and capillary system of concrete, than cause its expansion, since, in the latter case, the probability that all the ettringite formed in this study, from any origin: from $\mathrm{Al}_{2} \mathrm{O}_{3} \mathrm{r}^{-}$of $\mathrm{MK}$ and from $\mathrm{C}_{3} \mathrm{~A}$ of $P C$, precipitates in front of some aggregate particle is definitely smaller.

\section{CONCLUSIONS}

1. It has been shown that regardless of the analytic technique, test method and/or parameter considered, joint precipitation (co-precipitation) in the same plasterbearing solution, cement concrete or mortar, of ettringite from the $\mathrm{Al}_{2} \mathrm{O}_{3}{ }^{r-}$ origin, present in pozzolans, and ettringite from the $C_{3} A$ origin present in $O P C$, is always quantitatively speaking, more synergic than additive.

2. Depending on the parameter considered and from the technological standpoint only, the consequences of Expansive Synergic Effect, ESE, between the two types of ettringites can be esteemed to be beneficial, adverse or indifferent. But such judgements may not hold where two or more related parameters are 
a un mismo tiempo, los derivados de dos o más parámetros determinados más o menos afines. Así y desde el conocido y verificado comportamiento de los CPO, el de sus PUZC con $\mathrm{MK}$ y $7,0 \% \mathrm{SO}_{3}$, se puede calificar de:

- beneficioso, dados sus apropiadas resistencias mecánicas $y$,

- adverso, dado que sus estabilidades de volumen frente al ataque del yeso, no han sido constantes desde el principio al fin del ensayo (el $\Delta \mathrm{L}(\%)$ de sus probetas ASTM C 452-68 y de sus probetas de hormigón en función del tiempo, ha sido muy variable).

Por lo que en definitiva, se he da calificar necesariamente de adverso, puesto que este último comportamiento es el que prevalece en este caso sobre todo los demás.

Sin embargo, desde el conocido y verificado comportamiento de los "cementos hidráulicos expansivos" (51), el de muchos de estos PUZC con MK - familias P-1/MK, PY4/MK y PY-6/MK - ha sido beneficioso. Puesto que sus respectivos $\Delta \mathrm{L}_{7 \mathrm{~d}}(\%)$ estuvieron siempre comprendidos entre $0,04 \%$ y $0,10 \%$ (51); el resto -la familia PUZC P$2 / \mathrm{MK}-$, no. $\mathrm{Y}$ si se tratara de los "cementos sin retracción", también, y con mayor razón todavía si cabe.

3. De acuerdo con los resultados obtenidos de estos PUZC con $\mathrm{MK}$, cuando fueron ensayados conforme al método ASTM C 452-68, el 7,0\% SO 3 (equivalente a 15,05\% de yeso) que se les incorporó, no se comportó como agresivo sino como "regulador de fraguado". De aquí que sus tiempos de fraguado, PF Y FF, y el incremento de resistencias mecánicas en función del tiempo de dichos morteros selenitosos (15), fueran similares a los de cualquier CP. En cambio, cuando la cantidad de yeso aportada fue tres veces mayor (caso del método RT-86: $\Delta \mathrm{L}(1)$ ) y dos veces mayor (caso del método L-A (12) ), se comportó como agresivo, y originan por ello una "ataque rápido del yeso" a los CPO P-1 y P-2 y a sus PUZC con MK. Y si su CP matriz es el PY-4 o el PY-6 (ambos son CPRS) con el MK, también. Lógicamente, la co-precipitación de ettringita de ambos orígenes, de la $\mathrm{Al}_{2} \mathrm{O}_{3}{ }^{\mathrm{r}-}$ del $\mathrm{MK}$ y del $\mathrm{C}_{3} \mathrm{~A}$ de los $\mathrm{CPO}$, estuvo implicada en ambos comportamientos observados: el beneficioso y el adverso o perjudicial.

Por consiguiente, la adición de menores proporciones de yeso se puede considerar apropiada para algunos PUZC con MK, puesto que todos o sólo algunos de tales PUZC, con el contenido de yeso del método ASTM C 452-68 (= 15,05\% $=7,0 \% \mathrm{SO}_{3}(15)$ ), pueden ser catalogados como "cementos hidráulicos expansivos" (51), ya que dicha cantidad de yeso se comporta como "regulador de fraguado". En cambio, la adición de mayores cantidades de yeso, conduce a un ataque severo del yeso a los CP P-1 y P-2, y además rápido a sus PUZC, y a los CPRS PY-4 y PY-6 con MK también. considered at the same time.Thus and from the known and verified behavior of $O P C$, the performance of all these $\mathrm{POZC}$ with $\mathrm{MK}+7.0 \% \mathrm{SO}_{3}$, has been beneficial, according to mechanical strengths values, and adverse, according to the volume stability values due to that $\Delta L(\%) v s$. time has been greater and faster than the plain SRPC PY-6; nonetheless, their resulting final performances have been adverse.

However, from the known and verified behavior of "expansive hydraulic cements" (51) (whose use is specific, and for their controlled expansivity precisely, caution is needed when they are used), the performance of many POZC with MK-POZC families $P-1 / M K, P Y-4 / M K$ and $P Y-$ $6 / M K$ - has once again been beneficial, inasmuch as their respective $\Delta L_{7 d}(\%)$ were between $0.04 \%$ and $0.10 \%$ (51); the rest $-P O Z C$ family $P-2 / M K-$, can not. And if cements without drying shrinkage were considered, as well, and with much more reason yet.

3. According to ASTM C 452-68 test results, when $7.0 \%$ $\mathrm{SO}_{3}$, equivalent to $15.05 \%$ of gypsum, was added to each M pozzolan-containing Portland cement, it did not behave aggressively but rather as "setting regulator"; consequently, the increase in mechanical strengths versus time and times of setting were similar to the pattern observed in any PC. However, when the gypsum content was raised to slightly more than double or triple the proportion mentioned (33.33\% for the L-A test (12), and $45.16 \%$ for the $R T-86: \Delta L$ test (1)), it behaved aggressively. Logically, the joint precipitation of ettringite from $\mathrm{Al}_{2} \mathrm{O}_{3}{ }^{r-}$ and $\mathrm{C}_{3} \mathrm{~A}$ origins, were involved in the beneficial and adverse behavior observed.

Therefore the addition of the lower proportion of gypsum may be regarded as being suitable for some MK-containing POZC, insofar as all or most of these POZC meet the ASTM C 452-68 Standard $\left(=15.05 \%=7.0 \% \mathrm{SO}_{3}(15)\right)$ criteria for "expansive hydraulic cements" (51). Addition of higher amounts, on the contrary, leads to an aggressive and fast gypsum attack on OPC $P-1$ and $P-2$ and their POZC, and on the SRPC PY-4 and PY-6 with MK as well. 
4. Los diferente parámetros físicos, químicos y de resistencias mecánicas considerados han demostrado para mejor o para peor, que la ESE generada por la ettringita de origen alúmina reactiva, $0 \mathrm{Al}_{2} \mathrm{O}_{3}{ }^{\mathrm{r}}$, presente en puzolanas, y la de origen $\mathrm{C}_{3} \mathrm{~A}$ presente en $\mathrm{CPO}$, cuando ambas co-precipitan en un medio selenitoso común, ha sido siempre promovida por la alúmina reactiva o alúmina amorfa en este caso, presente en el MK de esta investigación.

Lógicamente, si dicha $\mathrm{Al}_{2} \mathrm{O}_{3}{ }^{\mathrm{r}}$ - hubiera sido primordialmente vítrea, caso de las cenizas volantes, las implicaciones prácticas derivadas por el ESE que originasen pueden verse algo retrasadas, pero no por mucho tiempo. Porque sea cual fuere la puzolana natural o artificial -ya sea ésta sub-producto industrial (ceniza volante) o no-, que se considere, toda o la mayor parte de su actividad puzolánica debe hacerse efectiva mecánicamente, a la edad de 28 días a lo sumo (54-56). Y en el caso de la construcción de pavimentos rígidos para carreteras de tráfico pesado, no más tarde de los 90 días (57).
4. The different physical, chemical and mechanical strength parameters used in this study, demonstrate, either for a good or for a bad cause, that the ESE generated by the ettringite from reactive alumina origin, $\mathrm{Al}_{2} \mathrm{O}_{3}{ }^{r-}$, present in pozzolans, and from $\mathrm{C}_{3} \mathrm{~A}$ origin, present in OPC, whether they co-precipitate in a common selenitous medium, is always promoted by the reactive alumina, $\mathrm{Al}_{2} \mathrm{O}_{3}{ }^{r-}$, or amorphous alumina present in the $M K$ of this research.

Logically, whether the mentioned $\mathrm{Al}_{2} \mathrm{O}_{3}{ }^{r-}$ was originally vitreous, case of fly ash, the practical repercussion derived from the ESE and caused due to its action, may be delayed, however, not for a long time. Therefore, regardless of the considered pozzolan type, natural or artificial, being industrial sub-product as fly ash, or not, the whole or the major part of its pozzolanic activity has to be mechanically effective until at the most 28 daysaged (54-56), except for the case of rigid pavements, constructed for heavy traffic especially, where the pozzolanic activity of the fly ash applied, has to be efficient before 90 days-aged (57).

\section{BIBLIOGRAFÍA / BIBLIOGRAPHY}

(1) Talero, R.: "Contribution to the Analytical and Physical-Chemical Study of the System: Pozozlanic Cements-Gypsum-Water (200 \pm $\left.3^{\circ} \mathrm{C}\right) "$, Ph.D. thesis, Madrid, Madrid Complutensis University, Spain, 20th nov. 1986.

(2) Talero, R.: "Ettringite from Portland cement origin and ettringite from pozzolan origin: Analogies, differences and semiquantitative relation with their respective origins. Interrogation". 9th Intern. Congress on the Chemistry of Cement. Proceedings, vol. III, Theme II, pp. 93-100; New Delhi-India, nov. 1992.

(3) Talero, R.: "Comparative XRD analysis ettringite originating form pozzolan and form Portland cement". Cem. Concr. Res., vol. 26 (1996), no 8, pp. 1277-1283.

(4) Talero, R.: "Kinetochemical and morphological differentiation of ettringites by the Le Chatelier-Ansttet test". Cem. Concr. Res., vol. 32 (2002), pp. 707-717. doi:10,1016/S0008-8846(01)00749-9

(5) Talero, R.: "Kinetochemical and morphological differentiation of ettringites by metakaolin, Portland cements and the Le Chatelier-Ansttet test. Parameter: Vicat Needle Penetration". Silicates Industriels, vol. 68, no 11-12 (2003), pp. 137-146.

(6) Talero, R.: "Performance of the metakaolin and Portland cements forming ettringite: Kinetic and morphological differences". 11th Intern. Congress on the Chemistry of Cement, Proceedings, vol. 2, pp.853-867, Durban-South Africa, 11-16 may 2003.

(7) Talero, R.: "Performance of metakaolin and portland cements in ettringite formation as determined by ASTM C 452-68: kinetic and morphological differences". Cem. Concr. Res., vol. 32 (2005), pp. 1269-1284. doi:10,1016/j.cemconres.2004,10,002

(8) Talero, R.: "Performance of metakaolin and portland cements in ettringite formation as determined by Le Chatelier-Ansttet test: Kinetic and morphological differences and new specification". Silicates Industriels, vol. 72, no 11-12 (2007), pp. 191-204.

(9) Talero, R.: "Kinetic and morphological differentiation of ettringites by metakaolín, Portland cements and ASTM C 452-68 test. Part I: Kinetic differentiation". Mater. Construcc., vol. 58, no 292 (2008), pp. 45-68.

(10) Talero, R.: "Kinetic and morphological differentiation of ettringites by metakaolin, portland cements and ASTM C 452-68 test. Part II: Morphological differentiation by SEM and XRD analysis". Mater. Construcc., vol. 59, no 293 (2009), pp. 17-34.

(11) Rahhal, V.; Irassar, E..; Trusilewicz, L.; Pedrajas, C.; Talero, R.: "Ettringite formation from pozozlan origin at early ages". Construction and Building Materials, Ref. no: CONBUILDMAT-D-11-00166 (en evaluación).

(12) Blondiau, L.: "Considérations diverses relatives à l'essai de résistance chimique au sulfate de calcium suivant le processus Le Chatelier-Ansttet". Rev. Mat. Constr. Trav. Publics. III, no 546 (1961), pp. 189-200.

(13) Talero, R.: "Sulphate resistance (high and moderated) of Portland cements. Accelerated tests: Specifications", Monograph no .399; Insto.C.C."Eduardo Torroja"-CSIC, C/ Serrano Galvache no. 4, 28033-MADRID-Spain, dec. 1989.

(14) Talero, R.; Bollati, M. R.; Hernández-O, F.: "Manufacturing non-traditional mortars and concretes by Portland cement, metakaolin and gypsum (15,05\%)". Mater. Construcc., vol. 49, no 256 (1999), pp. 29-41. doi:10,3989/mc.1999.v49.i256,433 
Co-precipitación de ettringita de rápida y lenta formación. Consecuencia: Efecto Sinérgico Expansivo. Su demostración mediante morteros y hormigones Co-precipitation of ettringite of rapid and slow formation. Consequence: Expansive Synergic Effect. Its demonstration by mortars and concretes

(15) ASTM C 452-68 Standard. Standard Test Method for potential expansion of portland cement mortars exposed to sulfate. Annual Book of Astm Standards. Part 9, Cement; Lime; Gypsum, pp. 298-300, 1968.

(16) Sanz, J.; Madani, A.; Serratosa, J. M; Moya, L. S.; Aza, S.: "Aluminum-27 and silicon-29 magic angle spinning nuclear magnetic resonance study of the kaolinite-mullite transformation". J. Am. Ceram. Soc., vol. 71, no [10] (1988), pp. C418-C421.

(17) Mejía, R.; Talero, R.: "Chlorides absorption and penetration into cement mortars with pozzolanic additions". 5th Iberoamerican Congress on Corrosion and Protection, Proceedings, Tenerife-Spain, 22-27 oct. 1995.

(18) Mejía, R.: "Contribution to the Analytical and Physical-Chemistry of the System: Portland Cements-Pozzolans-GBBS-Chloride-Water (at $\left.20 \pm 3^{\circ} \mathrm{C}\right)^{\prime \prime}$. PhD Thesis, Ftad. CC. Químicas, Universidad Complutense de Madrid-Spain, May 29th 1997 ("in Spanish").

(19) Mejía, R.; Delvasto, S.; Talero, R.: "A new pozzolan for high performance cementitious materials, Mater. Construcc., vol. 50, nº 260 (2000), pp. 5-13.

(20) Mejía, R.; Delvasto, S.; Talero, R.: "Chloride Portland measured by a modified permeability test in normal and blended cements". Advances in Cement Research, vol. 15, n० 3 (2003), pp. 113-118. doi:10,1680/adcr.2003,15,3,113

(21) Jones, M. R.; Mcphee, D. E.; Chudek, L. A.; Hunter, G.; Lannegrand, R.; Talero, R.; Scrimgeour, S. N.: "Studies using 27Al MAS NMR of AFm and AFt phases and the formation of Friedel's salt". Cem. Concr. Res., vol. 33 (2003), pp. 177-182. doi:10,1016/S00088846(02)00901-8

(22) Rahhal, V.: "Characterization of Pozzolanic Additions by Conduction Calorimetry", PhD Thesis, Universidad Politécnica de MadridSpain, dec.12th 2002.

(23) Rahhal, V. F.; Cabrera, O.; Talero, R.; Delgado, A.: "Calorimetry of Portland cement with silica fume and gypsum additions". J. Therm. Anal. Cal., vol. 87, no 2 (2007), pp. 331-336. doi:10,1007/s10973-005-7324-1

(24) Talero, R.; Rahhal, V.: "Influence of aluminic pozzolans, quartz and gypsum additions on Portland cement hydration". 12th Intern. Congress on the Chemistry of Cement. Proceedings, Montreal-Canada, 8-13 july 2007.

(25) Rahhal, V.; Talero, R.: "Calorimetry of portland cement with metakaolins, quartz and gypsum addtions". J. Therm. Anal. Cal., vol. 91, no 3 (2008), pp. 825-834. doi:10,1007/s10973-006-8250-6

(26) Talero, R.; Rahhal, V.: "Calorimetric comparison of portland cement containing silica fume and metakaolin: Is silica fume, like metakaolin, characterized by pozzolanic activity that is more specific than generic?". J. Therm. Anal. Cal., vol. 96, no 2 (2009), pp. $383-393$. doi:10,1007/s10973-008-9096-x

(27) Rahhal, V.; Talero, R.: "Calorimetry of Portland cement with silica fume, diatomite and quartz additions". Construction \& Building Materials, vol. 23 (2009), pp. 3367-374. doi:10,1016/j.conbuildmat.2009.06,003

(28) Rahhal, V.; Talero, R.: "Effect of three natural pozzolans on portland cement hydration". Mater. Construcc., vol. 53, n 269 (2003), pp. 29-40. doi:10,3989/mc.2003,v53,i269.266

(29) Rahhal, V.; Talero, R.: "Influence of two different fly ashes on the hydration of portland cements". J. Therm. Anal. Cal., vol. 78 (2004), pp. 191-205. doi:10,1023/B:JTAN.0000042167,46181,17

(30) Rahhal, V.; Talero, R.: "Fast physics-chemical and calorimetric characterization of fly ash". J. Therm. Anal. Cal., vol. 96, nº 2 (2009), pp. 369-374. doi:10,1007/s10973-008-9034-y

(31) Rahhal, V.; Talero, R.: "Fast physics-chemical and calorimetric characterization of natural pozzolans and other aspects". J. Therm. Anal. Cal., vol. 99, no 2 (2010), pp. 479-486. doi:10,1007/s10973-009-0016-5

(32) Rahhal, V.; Bonavetti, V.; Delgado, A.; Pedrajas, C.; Talero, R.: "Scheme of the Portland Cement Hydration with Crystalline Mineral Admixtures and Other Aspects". Silicates Industriels, vol. 74, no 11-12 (2009), pp. 347-352.

(33) Rahhal, V.; Cabrera, O.; Delgado, A.; Pedrajas, C.; Talero, R.: "C 4 AF ettringita and calorific synergic effect contribution". J. Therm. Anal. Cal., vol. 100, no 1 (2010), pp. 57-63. doi:10,1007/s10973-009-0204-3

(34) Eitel, W.: "Recent investigations of the system: lime-alumina-calcium-sulfate-water and its importance in building research problems". J. Am. Concr. Inst., vol. 28 (7) (1957), pp. 679-798.

(35) ASTM C 595M Standard: Standard Specification for Blended Hydraulic Cements.- ANNUAL BOOK OF ASTM STANDARDS, Section 4 Construction, vol. 04,01 Cement; Lime; Gypsum, pp. 291-296, 1995.

(36) Matousek, M.; Sauman, Z.: "Contribution to the hydration of expansive cement on the basis of metakaolinite". Cem. Concr. Res., vol. 4, no (1) (1974), pp. 113-122.

(37) Matuosek, M.; Sauman, Z.: A reply to P.K.Metha's and Chatterji's discussion on "Contribution to the hydration of expansive cement on the basis of the metakaolinite". Cem. Concr. Res., vol. 4 (1974), pp. 687-688.

(38) Metha, P. K.: A discussion of the paper "Contribution to the hydration of expansive cement on the basis of metakaolinite" by M. Matousek and Z. Sauman. Cem. Concr. Res., vol. 4 (1974), pp. 683-684.

(39) Chatterji, S.: A discussion of the paper "Contribution to the hydration of expansive cement on the basis of metakaolinite" by M. Matousek and Z. Sauman. Cem. Concr. Res., vol. 4 (1974), pp. 687-688.

(40) Silva, P. S. de; Glasser, F.P.: "Phase relation in the system CaO-Al2O3-SiO2-H2O relevant to metakaolin - Calcium hydroxide hydration". Cem. Concr. Res., vol. 23 (1993), pp. 627-639.

(41) Sabir, B. B.; Wild, S.; Bai, S. J.: "Metakaolin and calcined clays as pozzolans for concrete: a review". Cement \& Concrete Composites, vol. 23, no 6 (2001), pp. 441-454. doi:10,1016/S0958-9465(00)00092-5 
(42) Vu, D. D.; Stroeven, P.; Bui, B. V.: "Strength and durability aspects of calcined kaolin-blended portland cement mortar and concrete". Cement \& Concrete Composites, vol. 23, no 6 (2001), pp. 471-478. doi:10,1016/S0958-9465(00)00091-3

(43) Gruber, K. A.; Ramlochan, T.; Boddy, A.; Hooton, R. H.; Thoma, M. D. A.: "Increasing concrete durability with high-reactivity metakaolin". Cement \& Concrete Composites, vol. 23, no 6 (2001), pp. 479-484. doi:10,1016/S0958-9465(00)00097-4

(44) Dow, C.; Glasser, F. P.: "Álcali release from crushed minerals and thermally activated constituents of metakaolín". Advances in Cement Research, vol. 15, no 4 (2003), pp. 137-143. doi:10,1680/adcr.2003,15,4,137

(45) EN 196-5 Standard: Métodos de ensayo de cementos; Parte 5, Ensayo de puzolanicidad para cementos puzolánicos (Pozzolanicity test for POZCs or Frattini test).- AENOR, Calle Génova No. 6; 28004-MADRID-Spain ? Pliego de Prescripciones Técnicas Generales para la Recepción de Cementos RC-75 (Decreto de la Presidencia del Gobierno 1964/1975 de 23 de mayo - B.O.E. no 206 de 28 de agosto de 1975) = N. Frattini : Ann.Chim. Appl. 39 (1949), pp. 616-620.

(46) EN 196-3 Standard: Métodos de ensayo de cementos; Parte 3, Determinación del tiempo de fraguado (Times of Setting determination) y de la estabilidad de volumen (Le Chatelier's needles). AENOR.

(47) EN 196-1 Standard: Métodos de ensayo de cementos; Parte 1, Determinación de resistencias mecánicas (Mechanical Strengths determination). AENOR.

(48) Instrucción de Hormigón Estructural EHE, 1998 (R.D. 2661/1998, de 11 de diciembre). Secretaría General Técnica, Servicio de Publicaciones, Ministerio de Fomento, Po de la Castellana, Madrid, Spain.

(49) UNE 83308-86 and UNE 83308-93: Ensayos de hormigón. Determinación de la velocidad de propagación de los impulsos ultrasónicos. AENOR.

(50) Bollati, M. R.: "Ultrasound Energy: A new non-destructive test method for testing and designing concretes". $6^{\text {th }}$. Intern. Symp. on Concrete Roads, pp.8-10, 83-92; oct., 1990, Madrid-Spain.

(51) ASTM C 845 Standard: Standard Specification for Expansive Hydraulic Cement.- ANNUAL BOOK OF ASTM STANDARDS; Section 4 Construction, vol. 04,01 Cement; Lime ; Gypsum, pp. 390-393, 1990.

(52) Talero, R.: "Patología del hormigón. Ataque rápido del yeso: $2^{\mathrm{a}}$ Terapia preventiva. Óptimo de $\mathrm{SO}_{3}$ : su determinación". Ciclo de conferencias sobre: aplicaciones, patologías y terapias de estructuras, hormigón y otros materiales de contrucción, pp. 33-346; Confederación de Empresarios de la Construcción de Aragón, Cámara de Comercio e Industria de Zaragoza, 27 y 28 feb. y 1 de marzo de 1991.

(53) ASTM C 563 Standard: Standard Test method for Optimum $\mathrm{SO}_{3}$ in Hydraulic Cement Using 24-h Compressive Strength. Annual Book Of Astm Standards. Section 4 Construction, vol. 04,01 Cement; Lime; Gypsum, pp. 279-281, 1995.

(54) Instrucción para la Recepción de Cementos RC-08 (R.D. 956/2008 de 6 de junio; B.O.E. núm. 148, de 19 de junio de 2008.

(55) Pliego de Prescripciones Técnicas Generales para la recepción de Cementos RC-75 (Decreto de Presidencia del Gobierno 1964/1975 de 23 de mayo; BOE no. 206 de 28 de agosto de 1975).

(56) EN 450 Standard: Fly ashes as addition for concrete. Definitions, specifications and quality control. AENOR.

(57) PG3, Spanish Standard for Roads, 1994. Ministerio de Obras Públicas, Transportes, Comunicaciones y Medio Ambiente, Po de la Castellana, Madrid, Spain. 